\title{
Plateformes digitales et concurrence par la rapidité. Le cas des vêtements d'occasion
}

Recherche et Applications en Marketing

$1-24$

(C) l'Association Française du Marketing, 2021

Article reuse guidelines: sagepub.com/journals-permissions DOI: 10.1 I77/076737012199483 | journals.sagepub.com/home/ram

\section{Elodie Juge}

Univ. Lille, ULR 4999 - LUMEN, F-59000 Lille, France

\author{
Anissa Pomiès \\ emlyon business school, centre de recherche lifestyle, France
}

\section{Isabelle Collin-Lachaud (iD}

Univ. Lille, ULR 4999 - LUMEN, F-59000 Lille, France

\begin{abstract}
Résumé
Des recherches récentes explorent ce que les plateformes digitales font et font faire aux utilisateurs. Dans leur prolongement, cet article étudie comment les plateformes digitales P2P accélèrent les activités marchandes, et comment cette accélération modèle les relations entre utilisateurs. Une étude qualitative à caractère ethnographique a été menée au sein du marché des vêtements d'occasion de 2013 à 2020. En mobilisant la théorie de l'accélération sociale, nous montrons que les affordances des plateformes digitales encouragent une accélération du rythme de vie, une accélération du renouvellement des objets possédés, et une accélération technique multi-domaines. Ces formes d'accélération aboutissent à une concurrence par la rapidité entre utilisateurs : chacun d'eux se doit d'être plus réactif, plus à la pointe, et plus averti que les autres. De plus, les plateformes constituent un espace digital particulier qui invite à repenser l'aliénation sous plusieurs formes, et notamment l'aliénation par rapport à l'espace et par rapport au temps. Outre ses contributions théoriques, cette recherche offre des contributions sociétales visant à faire prendre conscience aux utilisateurs de l'ampleur de leurs activités marchandes accélérées sur les plateformes digitales.
\end{abstract}

\section{Mots-clés}

accélération, concurrence par la rapidité, contributions sociétales, P2P, plateformes digitales

\footnotetext{
Auteur correspondant :

Isabelle Collin-Lachaud, IAE Lille University School of Management, Avenue du Peuple Belge, 59000 Lille.

Email: Isabelle.collin-lachaud@univ-lille.fr
} 


\section{Introduction}

L'entrée dans le $21^{\text {ème }}$ siècle s'est accompagnée d'un dérèglement climatique et d'une hausse des inégalités ${ }^{1}$, conséquences d'un modèle capitaliste reposant sur la mise en concurrence des acteurs économiques et la surexploitation des ressources humaines et environnementales (Klein, 2019 ; Latour, 2015 ; Piketty 2013). Dans ce contexte, l'émergence des plateformes digitales a été perçue comme une alternative vertueuse à l'économie marchande traditionnelle (Botsman et Rogers 2010), notamment sur les plans environnemental (Chen et Kockelman, 2016) et social (Belk, 2014 ; Robert et al., 2014). Or, il semble que ces mêmes plateformes œuvrent aussi à rendre les échanges marchands entre utilisateurs de plus en plus rapides et faciles. Par exemple, dans le cas du vêtement d'occasion, les plateformes digitales P2P enjoignent les utilisateurs à être réactifs, leur expliquent comment vendre rapidement leurs articles, et leur imposent de courts délais d'expédition des articles vendus. Si l'on sait que les plateformes digitales P2P agissent sur le rythme des activités marchandes des individus (Denegri-Knott, 2011), on ne connait pas encore les effets de ces activités marchandes accélérées sur les relations entre utilisateurs. Cet article vient donc compléter la littérature en montrant comment les activités marchandes accélérées génèrent de la concurrence entre utilisateurs de plateformes P2P. Il est important d'analyser cette mise en concurrence des utilisateurs car, bien que la concurrence soit consubstantielle aux sociétés néolibérales, elle aliène les individus sans que ces derniers en aient conscience (Keucheyan, 2019). A travers cet enjeu d'objectivation et de dévoilement (Bourdieu, 1981), nous voulons aider les utilisateurs de plateformes à percevoir les mécanismes sociaux dans lesquels ils s'inscrivent afin qu'ils puissent agir de façon éclairée. In fine, nous souhaitons produire une connaissance ayant « un apport sociétal qui participe directement au bien-être de la société à court, moyen ou long terme » (Parguel et al., 2018 :14).

La littérature existante indique que les plateformes digitales agissent sur les utilisateurs (Benavent, 2016). Non seulement elles orientent leurs conduites (Kenney et Zysman, 2016), mais en plus elles contrôlent leur respect des règles du jeu (Möhlmann et Zalmanson, 2017), et modèlent la forme de leurs échanges marchands (Perren et Kozinets, 2018). Si les plateformes agissent sur les utilisateurs, elles offrent aussi à ces derniers un potentiel d'action via leurs fonctionnalités nommées « affordances 》 (Gibson, 1977 ; Light et al., 2018). Ainsi, les affordances rendent certaines actions possibles et en empêchent d'autres (Akrich et Latour, 1992). Si nous savons comment les plateformes digitales agissent sur les comportements individuels et les échanges, très peu de travaux explorent la façon dont elles accélèrent les activités marchandes. Seule Denegri-Knott (2011) aborde frontalement la question de la temporalité en montrant que les affordances accélèrent le cycle de désir chez le consommateur. L'auteure montre qu'en présentant continuellement de nouveaux biens désirables et en permettant leur acquisition rapide, les plateformes suscitent et annihilent en permanence les désirs du consommateur. Néanmoins, seule la relation utilisateur-objet désiré est explorée dans cet article, laissant dans l'ombre le réseau des utilisateurs. Notre article vise ainsi à combler ce manque dans la littérature en étudiant l'impact de l'accélération des activités marchandes générée par les affordances sur les relations entre utilisateurs.

Pour cela, nous avons réalisé une étude qualitative à caractère ethnographique au sein $\mathrm{du}$ marché des vêtements d'occasion entre mai 2013 et mai 2020. Pendant sept années, nous avons collecté des données par entretiens, observations et journal de bord que nous avons confrontées à la théorie de l'accélération sociale de Rosa (2012). Notre étude empirique montre que les plateformes digitales P2P alimentent plusieurs types d'accélération qui donnent lieu à une concurrence par la rapidité entre utilisateurs. En effet, un premier ensemble d'affordances génère une accélération du rythme de vie aboutissant à une concurrence pour être l'utilisateur le plus réactif. Un deuxième ensemble d'affordances encourage une accélération du renouvellement des objets possédés stimulant une concurrence pour être l'utilisateur le plus à la pointe. Un troisième ensemble d'affordances favorise une accélération technique multi-domaines donnant lieu à une concurrence pour être l'utilisateur le plus averti.

Notre recherche offre des contributions théoriques et sociétales. Sur le plan théorique, la mise en 
lumière du mécanisme de concurrence par la rapidité nous permet de contribuer à la littérature sur les plateformes digitales. Nous montrons qu'audelà de l'accélération du cycle de désir des utilisateurs individuels, les plateformes ont un impact plus large sur le réseau des utilisateurs. Leurs relations sont de nature compétitive, ce qui contrebalance les analyses existantes soulignant la formation de liens sociaux chaleureux entre utilisateurs de plateformes (Barraud de Lagerie et Sigalo Santos, 2018 ; Garcia-Bardidia, 2014). De plus, nous montrons que la concurrence entre utilisateurs constitue le moteur d'un système marchand auto-propulsé, au sens où la mise en mouvement des utilisateurs est alimentée par les plateformes elles-mêmes. Enfin, nous contribuons à la littérature sur l'accélération sociale en montrant que les plateformes constituent un espace digital particulier qui invite à repenser l'aliénation sous plusieurs formes, et notamment l'aliénation par rapport à l'espace et par rapport au temps. Notre recherche présente par ailleurs des contributions pratiques à visée sociétale. Nous invitons en effet les pouvoirs publics à contraindre les plateformes à ajouter de nouvelles affordances révélant aux utilisateurs l'ampleur de leurs activités marchandes accélérées et leur mise en concurrence.

Dans un premier temps, nous présentons les travaux sur les plateformes digitales $\mathrm{P} 2 \mathrm{P}$ et sur leurs affordances, puis nous introduisons la théorie de l'accélération sociale. Dans un deuxième temps, nous explicitons le design méthodologique à partir duquel les données ont été collectées et analysées. Dans un troisième temps, nous détaillons les résultats de notre étude de terrain. Enfin, dans un dernier temps, nous détaillons les contributions théoriques et sociétales de notre recherche.

\section{Revue de littérature}

\section{Des plateformes digitales marchandes agentiques}

Les plateformes digitales sont « des mixtures compliquées faites de logiciels, de matériels, d'opérations et de réseaux " grâce auxquelles se déroulent les échanges entre utilisateurs (Kenney et Zysman, 2016 : 64). Ces échanges peuvent prendre plusieurs formes comme la vente, la location, le prêt, le troc ou le don (Jacquet, 2015 ; Peugeot et al., 2015). Le type d'accès au bien ou au service échangé dépend de la présence ou de l'absence de compensation monétaire et de transfert de droits de propriété (Bardhi et Eckhardt, 2012). Les plateformes digitales se sont multipliées depuis la fin des années 2000 (Beauvisage et al., 2018 ; Decrop, 2017), et plus particulièrement celles qui intermédient des échanges marchands entre particuliers (Perren et Kozinets, 2018). L'essor de ces plateformes digitales marchandes P2P est particulièrement visible dans les secteurs de l'hébergement, du transport, de la mode, du loisir créatif, et des petits services (Beuscart et Flichy, 2018 ; Luckman, 2013 ; Zervas et al., 2017). A leurs débuts, ces plateformes ont été vues comme une alternative à l'économie traditionnelle pour leur impact environnemental positif et leur capacité à créer du lien social (Botsman et Rogers, 2010 ; Ertz et al, 2018). Néanmoins, les utilisateurs y ont recours principalement pour des raisons individuelles, utilitaristes et économiques (Bardhi et Eckhardt 2012 ; Padmavathy et al., 2019). En effet, l'activité marchande sur les plateformes fournit un complément de revenus aux offreurs (Barraud de Lagerie et Sigalo Santos, 2018 ; Beuscart et Flichy, 2018), permet aux utilisateurs malins de faire de bonnes affaires (Peugeot et al., 2015), et offre des opportunités de consommation inédites aux utilisateurs dont le pouvoir d'achat est limité (Benavent 2016 ; Robert et al., 2014).

Chaque plateforme est dotée d'une architecture qui oriente les flux d'informations et les activités des utilisateurs (Benavent, 2016). L'architecture des plateformes est indissociable de leurs affordances. Créé par Gibson (1977), le terme " affordance » renvoyait initialement aux propriétés matérielles d'un artefact suscitant des possibilités d'action (Leonardi, 2011). Dans sa réappropriation du concept, Norman (1988) a spécifié que le but d'une affordance est de signaler aux utilisateurs ce que les technologies peuvent faire et comment les individus peuvent réaliser leurs actions. La diffusion du concept a par la suite généré des débats et évolutions sémantiques (Parchoma, 2014). Pourtant, il existe aujourd'hui un consensus dans la littérature sur deux éléments majeurs. Tout d'abord, les affordances sont 
relationnelles (Leonardi et Vaast, 2017). Elles ne sont pas de simples propriétés matérielles des technologies ; elles existent dans la relation entre les individus et la matérialité des artefacts avec lesquels ils interagissent (Hutchby, 2001). Ensuite, les affordances sont des préconditions de l'activité (Greeno, 1994). Elles offrent un potentiel d'action aux utilisateurs (Leonardi et Vaast, 2017) ; elles empêchent certaines actions et en rendent d'autres possibles (Akrich et Latour, 1992). Elles sont donc à la fois des guides et des contraintes pour les utilisateurs (Bardini, 1996 ; Suthers, 2006). En marketing, le concept d'affordance est utilisé pour définir les fonctionnalités des plateformes qui soutiennent les possibilités d'action des utilisateurs (Denegri-Knott et Molesworth, 2013). Ainsi, s'intéresser aux affordances signifie accorder de l'attention à ce que l'architecture des plateformes permet et empêche de faire, et comment elle guide les actions qu'elle autorise (Light, Burgess et Duguay, 2018).

La littérature a mis en lumière les effets de la capacité à agir et à faire agir des plateformes digitales via leurs affordances. Tout d'abord, les affordances des plateformes digitales façonnent la forme de l'échange (Peugeot et al., 2015). Cette forme dépend du degré d'intermédiation de la plateforme et de l'intensité des interactions sociales entre utilisateurs qu'elles permettent (Perren et Kozinets, 2018). De plus, les plateformes digitales contrôlent les utilisateurs (Möhlmann et Zalmanson, 2017). Grâce à leurs systèmes de notation et de traçage d'activités, les plateformes peuvent surveiller les utilisateurs et exclure ceux qui enfreignent les règles du jeu qu'elles ont instaurées (Rosenblat et Stark, 2016). Enfin, les affordances des plateformes digitales soulagent les utilisateurs de l'investissement émotionnel et cognitif impliqué dans l'activité marchande (Denegri-Knott et Molesworth, 2013). Par exemple, sur eBay, les listes de souhaits, filtres et recommandations personnalisées diminuent les efforts en jeu dans la recherche d'objets à désirer (Denegri-Knott et Molesworth, 2013). Pour résumer, les affordances assurent le bon fonctionnement des plateformes en guidant les comportements individuels et en structurant la forme des échanges.

Si l'on sait que les plateformes digitales guident les comportements individuels, on sait encore peu de choses sur la manière dont elles modèlent la temporalité des activités marchandes. A notre connaissance, la seule recherche investiguant cette question est celle de Denegri-Knott (2011). L'auteure montre que les affordances d'eBay accélèrent le cycle de désir. En signalant en permanence de nouveaux éléments désirables, la technologie alimente la passion de consommer (Kozinets et al., 2017). La plateforme nourrit le culte de la nouveauté et initie en permanence de nouveaux désirs (Belk et al., 2003). Cependant, l'acquisition rapide de biens transforme la relation entre l'acheteur et l'objet : obtenir très rapidement ce que l'on désire diminue l'excitation suscitée par l'envie d'acquérir l'objet (Galluzzo, 2020). Paradoxalement, les plateformes achèvent tout aussi rapidement les cycles de désir qu'elles ont initiés, et poussent à en créer de nouveaux (Denegri-Knott, 2011). En explorant l'accélération du cycle de désir, Denegri-Knott (2011) prend pour unité d'analyse la dyade consommateur-objet désiré. Aussi instructive qu'elle soit, son analyse laisse de côté le réseau des utilisateurs. Notre objectif est de poursuivre la réflexion initiée par Denegri-Knott (2011) en étudiant les conséquences de l'accélération des activités marchandes sur le réseau des utilisateurs. Plus précisément, nous étudions comment les activités marchandes accélérées par les plateformes modèlent les relations entre utilisateurs. Pour cela, nous mobilisons la théorie de l'accélération sociale.

\section{Une société moderne accélérée}

Pour Rosa (2012), l'accélération sociale est une caractéristique de la modernité tardive et correspond à l'intrication de trois formes d'accélération. La première forme est l'accélération technique. Celle-ci désigne " l'accélération intentionnelle de processus orientés vers un but » (Rosa, 2012 : 18). Par exemple, dans le domaine de la production, cela revient à accroître le nombre d'objets produits par jour grâce à la mise au point de nouvelles machines et à l'adoption de formes d'organisation $\mathrm{du}$ travail plus efficaces. La deuxième forme est l'accélération $d u$ changement social. Celle-ci désigne la rapidité croissante avec laquelle surviennent les changements au cours d'une vie. Cela consiste par exemple à changer souvent de partenaire dans sa vie privée ou d'emploi dans sa 
vie professionnelle. La troisième forme est l'accélération du rythme de vie. Celle-ci est définie comme la tendance à « comprimer les actions et les expériences » (Rosa, 2012:27). Cette augmentation du nombre d'actions par unité de temps consiste à raccourcir les pauses entre deux tâches ou à réaliser plusieurs tâches en même temps.

D'après Rosa $(2012$; 2017), les individus de la modernité tardive sont dans une lutte permanente pour maintenir leur position dans l'espace social. Ils doivent déployer de plus en plus d'énergie pour rester compétitifs et être reconnus socialement. La position sociale étant désormais indexée sur les accomplissements, ces derniers doivent être sans cesse répétés afin que l'individu conserve sa place dans le monde (Rosa, 2017). S'arrêter conduisant inéluctablement à être dépassé, chacun est forcé à être toujours plus rapide s'il veut rester dans la course (Rosa, 2017). La logique sociale de la compétition génère une lutte quotidienne faisant triompher les rapides aux dépends des lents (Rosa, 2012). Ce phénomène d'accélération sociale régit les comportements individuels dans de nombreuses sphères notamment professionnelle, personnelle, politique et marchande.

Cette accélération généralisée engendre plusieurs conséquences. La première est l'apparition de situations de désynchronisation (Rosa, 2012). C'est par exemple le cas lorsque les êtres humains extraient des ressources naturelles à une vitesse supérieure à celle de leur renouvellement, ou lorsqu'un individu est en surrégime et travaille avec une intensité supérieure à celle que son corps peut soutenir. Ces cas de désynchronisation sont sources de frictions temporelles et génèrent un ensemble de troubles comme l'épuisement, la confusion et le stress (Husemann et Eckhardt, 2018 ; Rosa, 2012). L'autre conséquence de l'accélération sociale est l'aliénation, définie comme une modification du rapport au monde qui favorise les rapports muets et froids aux dépends de rapports signifiants et résonants (Rosa, 2017). Rosa (2012) identifie cinq formes d'aliénation. L'aliénation par rapport aux choses renvoie à un mode de relation aux objets matériels où ceux-ci demeurent extérieurs, détachés, et non responsifs du fait d'une tendance à s'en débarrasser rapidement sans se les approprier. L'aliénation par rapport aux actions désigne notre tendance à oublier ce que nous souhaitons réellement faire, et à réaliser à la place des actions que nous nous sentons contraints de faire (ex. : aller au bout de la to do list) et « des activités de consommation à gratification immédiate » (Rosa, 2012 : 127). L'aliénation par rapport au temps a trait à notre propension à cumuler une série d'expériences déconnectées les unes des autres (ex : sortir du travail, écouter un podcast dans le métro, assister à une pièce de théâtre, appeler un proche dans les transports, etc.). L'aliénation par rapport aux autres renvoie quant à elle à notre tendance à rencontrer une foule de personnes sans pour autant construire de relations intenses et significatives avec elles. Enfin, l'aliénation par rapport à l'espace désigne un désengagement, un désinvestissement du lieu physique ou matériel dans lequel nous évoluons du fait d'une mobilité accrue. Notre recherche complète Rosa (2012) en étudiant les pratiques marchandes accélérées sur les plateformes afin de comprendre comment se manifestent les différentes formes d'aliénation et en particulier l'aliénation par rapport à l'espace, lorsque ce dernier est digital. Ainsi, à l'issue de cette revue de littérature, nous posons les questions de recherche suivantes : Comment les plateformes digitales contribuent-elles à l'accélération des activités marchandes? Comment ces activités marchandes accélérées modèlent-elles les relations entre utilisateurs?

\section{Contexte et méthodologie}

Pour répondre à nos questions de recherche, nous avons choisi d'explorer un marché majeur, celui des vêtements d'occasion en France. L'intérêt croissant des Français pour les vêtements d'occasion et les enjeux économiques qui découlent de l'essor des plateformes digitales de vide-dressing expliquent le choix de notre contexte d'étude. Ce marché était estimé à un milliard d'euros en 2018, et cette tendance est à la hausse selon l'Institut Français de la Mode. Ainsi, 39\% des Français déclarent avoir acheté un vêtement d'occasion en 2019 et 48\% indiquent vouloir en acheter davantage en $2020^{2}$. Cet engouement est nourri par l'essor des plateformes digitales qui fluidifie les échanges (Benavent, 2016), offrant ainsi aux producteurs un accès direct à leurs clients et permettant à des 
particuliers d'acheter et de vendre à d'autres particuliers (Hamari et al., 2015). Les premières plateformes digitales spécialisées en vêtement d'occasion sont apparues en 2008 et connaissent depuis lors un succès croissant. A titre d'exemple, Vinted - le leader sur le marché - gagne 23000 nouveaux utilisateurs par jour en France pour un total de 10 millions $^{3,4}$. Plus de 400000 articles sont mis en ligne chaque jour ${ }^{5}$, pour un total de 120 millions d'articles ${ }^{6}$.

Pour investiguer ce contexte empirique, nous avons réalisé une étude qualitative à caractère ethnographique de mai 2013 à mai 2020. Tout au long de ces sept années, plusieurs méthodes ont été mobilisées conjointement (voir Annexe 1). Nous avons conduit 19 entretiens individuels en profondeur auprès de pratiquantes de vide-dressing ( 9 entretiens en 2013, 2 en 2014, 2 en 2016, 2 en 2017 et 4 en 2019). Etaler les entretiens sur un temps long a permis d'ajuster mutuellement notre question de recherche et notre collecte de données comme cela est prescrit par Arsel (2017). La pratique de vide-dressing en ligne attirant très majoritairement des femmes ${ }^{7}$ jeunes ( $«$ Les utilisateurs sont majoritairement des femmes, et près de $60 \%$ d'entre eux ont entre 18 et 29 ans $»^{8}$ ), nous avons recruté des personnes dont le profil sociodémographique reflète celui de la grande majorité des individus engagés dans la pratique (Maciel et Wallendorf, 2017) (voir Annexe 2). Le recrutement a mêlé opportunisme et technique de boule de neige (Royer et Zarlowski, 2007). Dans le lieu public de leur choix (café, bar, fast-food), les utilisatrices ont été interrogées sur leur définition du vide-dressing en ligne (perceptions et représentations), sur leurs débuts dans cette activité marchande (motivations, difficultés, et pratiques initiales), sur leurs pratiques actuelles (modalités de l'activité, relation avec les autres utilisatrices, rapport aux plateformes), et sur leur vestiaire (nombre et types d'articles, marques, gestion du contenu). Chacune des utilisatrices étant en possession de son téléphone portable pendant l'entretien, elle a pu nous montrer sa façon d'utiliser les plateformes. Les entretiens ont duré entre 62 et 94 minutes pour une durée moyenne de 67 minutes, ce qui représente au total $21 \mathrm{~h} 30$ d'enregistrement et 140 pages de retranscription.

Nous avons complété les entretiens avec des observations en ligne sur trois plateformes de vide-dressing : Vide Dressing, Vestiaire Collective et Vinted. Deux raisons ont motivé la sélection de ces plateformes. Non seulement ce sont les plateformes évoquées par les répondantes lors des premiers entretiens, mais en plus elles correspondent aux caractéristiques énoncées par Bernard (2004) : (1) des communautés larges, (2) très actives spécialisées dans la vente de vêtements et d'accessoires d'occasion et (3) animées par un noyau dur de membres. Menées sur les sites web et sur les applications mobiles, les observations en ligne avaient trois objectifs. Tout d'abord, elles nous ont permis de nous acculturer à la pratique et donc de rebondir pertinemment pendant les entretiens. Ensuite, elles ont permis de générer de la diversité dans nos données pour contrebalancer l'homogénéité du profil des pratiquantes interviewées (Barraud de Lagerie et Sigalos Santos, 2018). Pour cela, nous avons prêté attention au profil des utilisateurs (ex. : genre, taille, région, pays) et aux spécificités de leurs pratiques (nombre d'articles à vendre et/ou déjà vendus, prix de vente, évaluations, nombre d'abonnés et nombre d'abonnements, et heure de la dernière connexion). Enfin, les observations en ligne ont permis d'analyser les affordances des plateformes. Pour cela, nous avons créé un compte d'utilisateur et navigué sur les plateformes en tant qu'acheteur et vendeur, explorant ainsi les interfaces à la manière de la "walkthrough method" (Light et al., 2018). Nous avons prêté attention à ce que les fonctionnalités des plateformes encouragent, forcent, ou empêchent de faire. Concrètement, nous avons identifié les modalités d'inscription, le nombre et la position des boutons et icônes, le contenu des profils d'utilisateurs, l'arborescence des sites, les messageries, les notifications, les pop-ups, les champs obligatoires, les conditions générales de vente, les modalités de paiement et de livraison proposées (Light et al., 2018). Au total, nous avons navigué sur ces plateformes pendant plus de 550 heures entre 2013 et 2020 et produit 230 captures d'écran.

Un journal de bord a été tenu pendant les sept années de la recherche. De nombreux éléments ont été méticuleusement consignés selon la méthodologie proposée par Groleau (2003). Ce journal de bord comporte 107 pages et regroupe les notes d'observation en ligne, des mémos sur les utilisatrices, des verbatim de pratiquantes, et des 
analyses préliminaires. Ces éléments relatent le plus précisément possible les actions et interactions relevées lors des observations, mais aussi les relations informelles entretenues avec les informateurs privilégiés (Badot et al., 2009). Ce journal de bord a facilité la compréhension de l'objet d'étude tout en aidant à la prise de recul réflexif lors des phases de collecte et d'analyse de données (Allard-Poesi, 2015). Le recours conjoint à différentes méthodes de collecte a permis de constituer un corpus consistant et de procéder à une triangulation des données assurant la robustesse des résultats (Arnould et Price, 1993) (voir Annexe 3).

Nous avons analysé nos données selon un processus itératif de va et vient entre notre corpus complet et la littérature (Sandikci et Ger, 2010), comme requis dans les approches interprétatives (Arsel, 2017). Tout d'abord, nous avons commencé par une phase de « lecture flottante » afin de comprendre en profondeur les données (Dumez, 2013). Ensuite, nous avons démarré le processus de codage à visée théorique pour organiser et donner du sens à notre matériau empirique (Miles et Huberman, 1994 ; Point et Voynnet-Fourboul, 2006). Pour les entretiens, nous avons repéré des unités de texte homogènes du point de vue sémantique et les avons catégorisées, c'est-à-dire attribué un label (Spiggle, 1994). Pour les données d'observations, nous avons identifié toutes les affordances repérées tant dans les captures d'écran que dans le journal de bord, conservé celles qui apparaissent dans les trois plateformes étudiées, et regroupé en catégories d'analyse celles qui ont des rôles similaires. Pour les entretiens comme pour les observations, la phase de montée en abstraction a permis de passer de catégories empiriques à des construits plus analytiques (Spiggle, 1994). En confrontant la littérature sur l'accélération, nos entretiens et nos données d'observations, nous avons fait progressivement émerger des liens entre nos catégories d'analyse, et mis en évidence le mécanisme intégrateur de concurrence par la rapidité.

\section{Résultats}

Les affordances des plateformes étudiées favorisent trois formes d'accélération qui encouragent une concurrence par la rapidité entre utilisatrices.
Celle-ci se manifeste par trois injonctions : être la plus réactive, être la plus à la pointe, et être la plus avertie. Bien que ces trois injonctions soient empiriquement intriquées, nous les séparons analytiquement pour des raisons de clarté.

\section{Être la plus réactive}

Les plateformes digitales étudiées comportent des affordances qui favorisent l'accélération du rythme de vie. Tout d'abord, elles disposent de marqueurs temporels qui donnent des informations sur l'activité des utilisatrices. Par exemple, l'heure de la dernière connexion est spécifiée sur Vinted, tandis que l'heure à laquelle les messages sont envoyés est mentionnée sur Vide Dressing et Vestiaire Collective. Ces indications permettent de calculer le temps de réponse d'une utilisatrice, comme le montrent les notes de terrain suivantes :

Je clique sur son pseudo et lui envoie le message suivant (16H03) : "Bonjour, je trouve le pantalon Sézane que vous mettez en vente vraiment joli! J'ai une petite question à propos de la coupe : est-ce une coupe 7/8 qui s'arrête au-dessus de la cheville? Je vous remercie et vous souhaite une bonne journée. " [...] A 16h28, je reçois un mail de Vide Dressing intitulé «nouveau commentaire pour l'article Pantalon carotte Sézane ». Je consulte directement la page de l'annonce, et constate que la vendeuse m'a répondu : «Bonjour. Il s'agit en effet d'un 7/8. Du moins pour mon 1 m65. Je vous mets des photos portées pour mieux vous rendre compte. " Les messages postés par les acheteuses potentielles et la vendeuse sont visibles de tous car la messagerie Vide Dressing est publique. Chaque commentaire donne à voir le pseudo de celui ou celle qui l'écrit, la date et l'heure à laquelle il a été posté. Il s'est écoulé 25 minutes entre le moment où ma question a été publiée, et le moment où la vendeuse a publié sa réponse.

Les trois plateformes disposent aussi d'un mode « vacances » qui permet de désactiver les annonces, et d'éviter ainsi de fausser les indicateurs de réactivité des utilisatrices en cas d'absence. Ces marqueurs temporels suggèrent que la rapidité et la réactivité sont des critères pertinents pour définir les utilisatrices, et participent à leur qualification comme agents ayant une dimension temporelle 
(Callon et al., 2000). De plus, les plateformes imposent des conditions temporelles de validité aux transactions, ce qui force les utilisatrices à finaliser le plus vite possible leurs échanges. Vide Dressing contraint à envoyer les articles vendus en moins de trois jours sous peine d'annuler la transaction. Vinted impose quant à elle un délai de cinq jours, comme l'indique Charlotte (28 ans) : " Il y a une pression sur les envois. [...] Bah Vinted, il te conditionne pour que ça aille vite. Max j'ai 5 jours pour envoyer sinon la transaction est annulée. Moi, je fais le lendemain $\max »$. Les conditions temporelles de validité instaurent une pression temporelle situationnelle qui s'achève une fois le colis envoyé, mais qui se transforme en pression temporelle chronique lorsque les transactions marchandes sont répétées dans le temps (Gourmelen et al., 2016). Les conditions temporelles de validité s'appliquent également aux offres promotionnelles, toutes ayant une durée de vie limitée à quelques heures ou quelques jours seulement. En outre, les plateformes envoient en permanence des notifications via leur application et par mail. Ces notifications sont envoyées à plusieurs occasions : confirmer l'inscription, communiquer une offre promotionnelle, informer d'une nouvelle vente, alerter qu'un utilisateur a commencé à suivre votre profil (Illustration 1), et prévenir de la baisse d'un prix ou de la mise en vente d'un article désiré. Enfin, les plateformes diffusent un discours normatif dans lequel la veille constante est érigée comme clé du succès. Par exemple, la plateforme Vestiaire Collective spécifie les bons comportements pour augmenter ses chances de vendre : « soyez attentif » (en activant les notifications), « interagissez » (en répondant dès que possible aux questions) ou encore « soyez réactif» (en étant ouvert aux offres reçues).

Ces affordances contribuent à l'accélération du rythme de vie des utilisatrices, définie comme une augmentation de leur nombre d'actions par unité de temps (Rosa, 2012). En effet, les discours et les comportements observés montrent que les utilisatrices gardent en permanence un œil sur leur téléphone afin de vérifier l'arrivée de nouvelles notifications. Il en découle une intrication entre leur activité marchande et leur vie quotidienne. Les répondantes sont en mode multitâches permanent que ce soit au travail, à la maison, ou dans les transports. Julie (22 ans) explique : « Je suis dessus

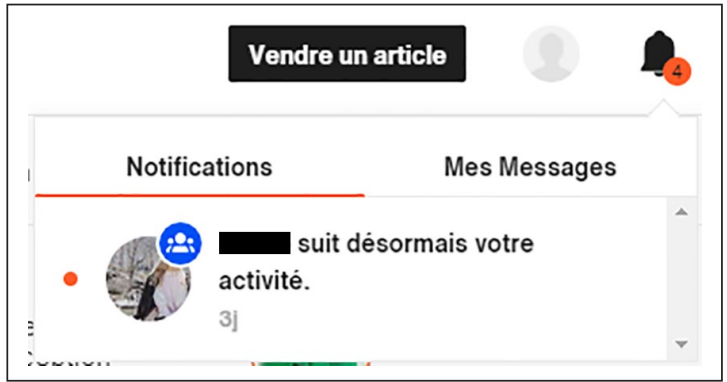

Illustration I. Notification reçue via la plateforme Vestiaire Collective.

tous les jours, en général 15 à 20 minutes le matin, en métro, et au petit café, et le soir après le journal de TF1, devant la télé dans le canapé. Le midi, je regarde et je montre aussi à mes collègues, je montre, je demande ce qu'elles en pensent ». Comme Julie, les utilisatrices interviewées insistent sur la dimension chronophage de l'activité de videdressing en ligne, et sur leur difficulté à se déconnecter des plateformes. Le rythme de l'activité de shopping en ligne s'insère donc complètement dans la temporalité des utilisatrices (MichaudTrévinal et Stenger, 2018). L'accès aux plateformes via les applications sur téléphone modifie l'allocation du temps des utilisatrices, leur efficacité sur les tâches menées en parallèle, et leur temps disponible (Lallement et Gourmelen, 2018). Certaines utilisatrices interviewées indiquent qu'elles procrastinent sur des tâches importantes non liées au vide-dressing en ligne. C'est par exemple le cas de Manon (25 ans) qui nous a confié ne pas avancer dans sa recherche d'un logement à acheter car toute son attention est dirigée vers ses activités de vente et d'achat de vêtements d'occasion. Ceci témoigne d'une aliénation des utilisatrices par rapport aux actions (Rosa, 2012), au sens où les activités marchandes à gratification immédiate occupent l'essentiel de leurs actions quotidiennes.

L'accélération du rythme de vie des utilisatrices donne lieu à une concurrence entre elles par la rapidité. Pour les vendeuses, il s'agit de réagir tout de suite aux notifications, d'être la plus rapide à répondre aux demandes des acheteuses, et d'être également plus rapide que la moyenne à envoyer les articles vendus. Les utilisatrices interviewées semblent avoir intériorisé cette concurrence par la 


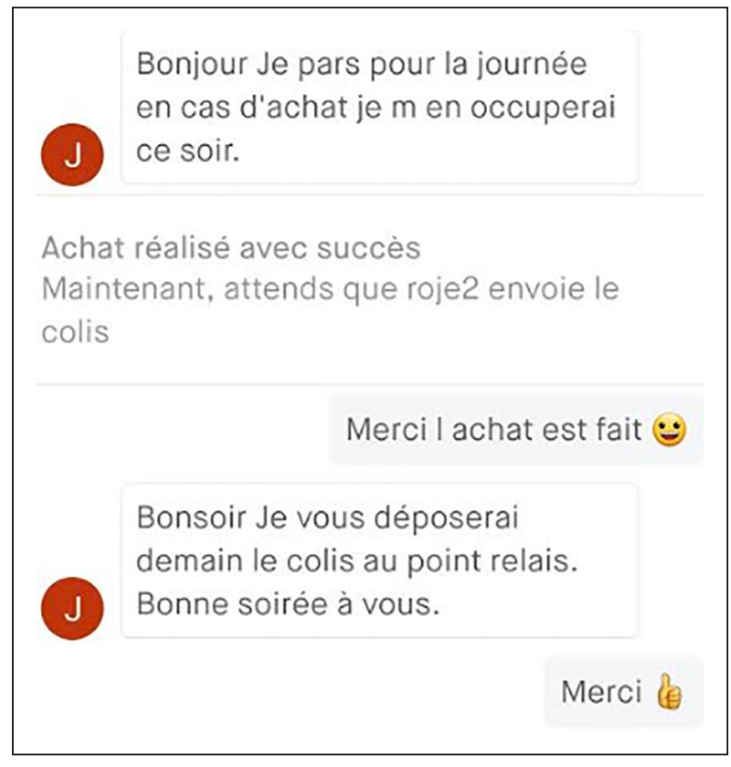

Illustration 2. Exemple d'échange marchand réalisé par un des auteurs sur Vinted.

rapidité et sanctionnent celles qui ne s'y conforment pas. Sandra (48 ans) explique : "Aujourd'hui, 3 jours ça me parait hyper long, quand une fille me fait une demande, je réponds hyper vite, je me doute qu'elle est pressée comme moi et puis pareil, j'envoie vite pour qu'elle le reçoive vite [. . .] Ça compte pour la note sur Vinted, si tu ne vas pas assez vite, les filles elles râlent et elles mettent des mauvaises notes ». En ce qui concerne les acheteuses, l'objectif primordial est d'être la plus rapide à « shopper la bonne affaire » (Inès, 22 ans). Nina (20 ans) souligne qu'elle achète dès qu'elle reçoit une réponse favorable de la vendeuse : " Je suis sur l'appli Vinted sur mon portable, je regarde vite fait sur le canapé le soir. J'envoie un message, la personne elle me répond et BAM j'achète!" . Pour acheter ou pour vendre, les utilisatrices cherchent à aller plus vite que les autres afin de ne pas rater une opportunité de transaction. Les affordances obligent les pratiquantes à une assiduité sans faille, et érigent la réactivité en clé du succès (Illustration 2).

\section{Être la plus à la pointe}

Les trois plateformes digitales étudiées présentent des affordances favorisant l'accélération du renouvellement des objets possédés. La première d'entre elles est l'interface intuitive. Il est très facile et rapide de créer un profil d'utilisateur - même sans compétences informatiques avancées - ce qui instaure de très faibles barrières à l'accès aux plateformes (Filser et al., 2019). Julie (22 ans) revient sur la facilité avec laquelle elle a créé son profil et ses annonces : "C C'est hyper pratique, quand j'ai commencé, j'ai tout publié et en 20 minutes c'était réglé. . C'est hyper pratique en deux trucs t'as mis ton annonce, c'est réglé. Il t'aide, il te guide ». Cette aisance est corroborée par les notes de terrain suivantes :

Je souhaite vendre sur Vestiaire Collective une paire de boucles d'oreilles que je ne porte plus. Je clique sur le gros bouton " vendre un article » et arrive sur une page qui me permet de créer une fiche-produit. [. . .] Les indications sont très claires, je n'ai pas à me poser de question. Par exemple, pour l'étape 1, je dois préciser la matière des boucles d'oreilles que je veux vendre et la couleur. La plateforme me donne le choix entre plusieurs options, je n'ai qu'à cocher ce qui se rapproche le plus de mes boucles. Il me faut 2 secondes pour trouver la matière « or jaune» et 2 secondes pour trouver la couleur « doré ».

A ceci s'ajoute la fluidité des transactions marchandes une fois le profil créé. En effet, l'achat ne nécessite que quelques clics grâce aux filtres affinant les requêtes. Les trois plateformes étudiées permettent de filtrer les résultats par genre, catégorie de produit, marque, taille, couleur, et prix. Cette simplicité du processus d'achat et de vente est accentuée par les tutoriels et les rubriques conseils qui constituent la deuxième affordance identifiée. Ces conseils clarifient les attentes pesant sur les photos des articles en vente (nombre, focus, netteté) et sur la manière de les décrire (marque, taille, matière, défauts potentiels). Par exemple, Vide dressing donne 10 conseils illustrés pour expliquer les bonnes pratiques en matière de photo (Illustration 3). Les tutoriels et conseils contribuent à standardiser les fiches descriptives et à créer un espace de calcul commun facilitant les comparaisons entre articles (Callon et Muniesa, 2003). Outre l'interface intuitive et les tutoriels, les plateformes étudiées comportent des marqueurs de nouveautés qui mettent en lumière les articles récemment mis en ligne. Vinted permet de trier les résultats des 


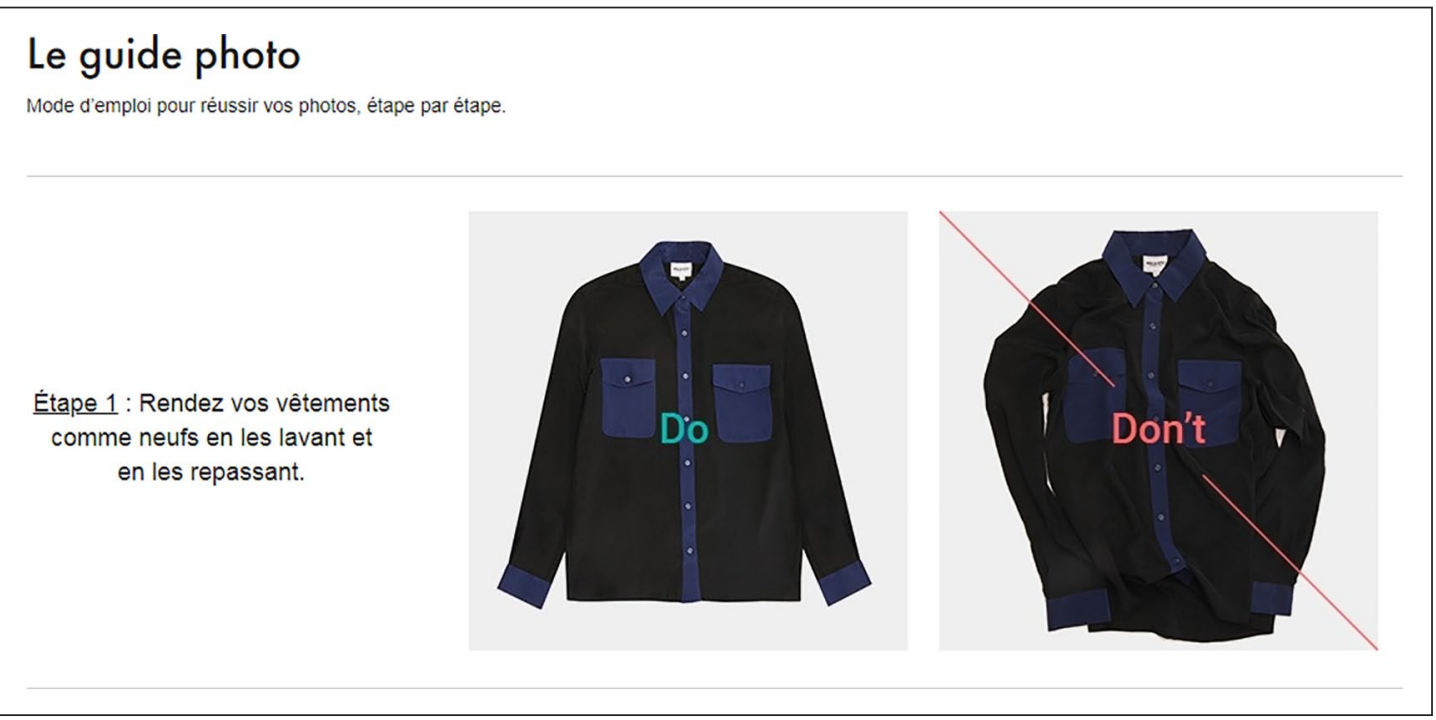

Illustration 3. Extrait du guide photo sur Vide Dressing.

requêtes du plus récent au moins récent; Vide Dressing a une page dédiée aux nouveautés, une sélection « nos derniers coups de cœur », et des pastilles « NEW ! » sur les annonces les plus récentes ; Vestiaire Collective a une page dédiée aux nouveautés et une section " nouveautés » par catégorie de produits. Les newsletters des trois plateformes contribuent elles aussi à mettre les nouveautés au premier plan. Enfin, les plateformes comportent des marques d'intérêt. Parmi elles se trouve la liste de souhaits, un espace virtuel dans lequel les utilisateurs placent les articles qui les tentent afin de les retrouver plus vite, de recevoir des nouvelles à leur sujet, et éventuellement de les acheter. La liste de souhaits permet également de pré-paramétrer des requêtes générant des recherches automatiques. Sur Vinted, nous pouvons lire : "Abonne-toi aux recherches - Tu peux t'abonner aux recherches et revenir plus tard pour vérifier s'il y a des nouvelles annonces qui correspondent à tes recherches ». Ainsi, si l'article n'est pas disponible immédiatement, les utilisateurs seront informés en temps réel dès qu'un article semblable sera disponible. Les trois plateformes spécifient par ailleurs le nombre d'utilisateurs ayant placé un produit dans leur liste de souhaits. Ceci permet d'évaluer le niveau d'urgence d'un achat potentiel : un article convoité par un grand nombre de personnes est un article qui peut être acquis rapidement par un acheteur concurrent.

Les affordances identifiées contribuent à l'accélération du renouvellement des articles possédés. Les entretiens révèlent que les utilisatrices ne sont pas dans une logique cumulative : elles n'augmentent pas le nombre de vêtements dont elles disposent. Par exemple, Nina (20 ans) souligne que les articles qu'elle achète sont destinés à être vendus peu après : "Les articles, ah ils ne restent pas longtemps dans mon placard, 4 ou 5 mois, après je sais que je vais plus les porter de toutes façons, et surtout, je sais que c'est quelque chose qui peut encore bien se vendre, encore à un prix convenable ». Les utilisatrices de plateformes ne diminuent pas non plus le nombre de vêtements qu'elles possèdent : elles vendent en vue d'acheter. Pour Ophélie (23 ans), vendre revient à libérer de l'espace dans son armoire afin d'accueillir de nouveaux articles : « Je vais faire de la place. . (rire) pour en acheter d'autres! (rire) ». En d'autres termes, les utilisatrices vendent et achètent afin de renouveler régulièrement leur stock de vêtements. Pour Manon (25 ans), les plateformes sont le moyen idéal de faire évoluer fréquemment sa garde-robe : « [le] plaisir de renouveler facilement ma garde-robe à moindre prix, oui la renouveler régulièrement! ". Comme Manon, Estelle (23 ans) cherche à « 
recycler [son] armoire rapidement » et Nina (20 ans) peut «changer de vêtements plus souvent ». Le renouvellement rapide de la garde-robe montre que les vêtements qui la composent passent rapidement de commodité à bien personnel avant de revenir à l'état de commodité ayant une valeur d'échange (Denegri-Knott et Molesworth, 2009 ; Kopytoff, 1986).

L'accélération du renouvellement des articles possédés induit une concurrence par la rapidité pour être la plus à la pointe. Ainsi, Inès (22 ans) indique qu'en renouvelant sa garde-robe via les plateformes, elle parvient à suivre les dernières tendances : «Je peux me faire plaisir et changer tout le temps de vêtement, être à la mode ». Claire (20 ans) partage le même souci d'être à la page : «Vider ses armoires, changer, en plus la mode elle change de toutes façons, il faut toujours être tip top, il faut être à la mode!». Les plateformes sont des alliées permettant de se caler sur les cycles courts de la mode, et de remplacer des objets dont l'obsolescence esthétique est un puissant moteur de consommation (Assouly, 2010). Certaines utilisatrices achètent des pièces sans les porter, simplement parce qu'elles sont à la mode et qu'elles pourront être aisément revendues à un prix plus élevé. Ces pièces constituent dès lors de simples « occasions de gains » (Chabault, 2020 : 94 ; Juge et al., 2019). La concurrence par la rapidité repose donc sur un art du décodage et de l'anticipation de tendances : il s'agit d'identifier ce qui est à la mode ou va bientôt l'être pour acheter l'article incontournable du moment ou qui le deviendra bientôt ; il s'agit aussi de repérer ce qui est démodé ou va bientôt l'être pour réussir à vendre un article avant qu'il ne trouve plus preneuse. Une conséquence de la concurrence pour actualiser le stock d'objets possédés est l'impossibilité de créer une relation résonante avec eux, ce qui génère une aliénation par rapport aux choses (Rosa, 2012). L'extrait de journal de bord écrit juste après l'entretien de Shéhérazade en 2013 fait état du détachement des utilisatrices vis-à-vis de leurs vêtements :

Les pratiquantes semblent comme "détachées » de leurs vêtements, elles prennent plaisir à acheter et vendre. Il n'y a pas d'attachement émotionnel - pas de j'adore ce pull - il me rappelle . . . non, juste - . . .ce pull vaut autant, la marque est prisée ... je peux le vendre à . . .

Les utilisatrices maintiennent les vêtements dans un état d'extériorité et de détachement que Rosa (2012) qualifie d'aliénation.

\section{Être la plus avertie}

Les trois plateformes digitales étudiées présentent des affordances favorisant l'accélération technique dans trois domaines : la communication, le transport, et le paiement. Dans le domaine de la communication, nous avons observé une évolution de la mise en contact vers des formats plus efficaces, et des contenus plus scriptés. En 2013, les utilisatrices rédigeaient entièrement des messages qu'elles échangeaient dans un espace privé (leur boite mail personnelle par exemple). Elles passaient alors beaucoup de temps à écrire et répondre aux messages d'autres utilisatrices. Léonie (24 ans) relate sa difficulté à gérer la profusion de mails et le risque de mauvaise évaluation qui pèse sur les utilisatrices lentes : «Je me souviens, dès le matin, une fois pour un sac à dos qu'ils vendaient chez [nom du magasin], je l'avais mis à 20€. Là-bas, il est vendu à 55€. Là j'ai eu au moins 200 mails, et puis il faut répondre! Parce que si tu ne réponds pas, t'es mal notée. . . Il y en a qui s'acharnent, si tu ne réponds pas tout de suite, elles te harcèlent de mails. Oui, ça prend du temps ». L'évolution des affordances a permis de résoudre ce problème et d'accélérer la communication entre utilisatrices via une mise en relation automatisée. Par exemple, sur Vestiaire Collective, en cliquant sur le bouton « faire une offre de prix », l'utilisatrice accède à une « salle de négociation » et se contente d'inscrire le prix auquel elle souhaite acheter l'article, éventuellement plus bas que celui indiqué sur l'annonce. La vendeuse est alors libre d'accepter ou de refuser la proposition, sans donner d'explication. Cette affordance existe aussi sur Vinted, comme le montrent l'illustration 4 et les notes de terrain suivantes :

Je décide de cliquer directement sur « faire une offre ». Un petit encadré apparait avec le chiffre « $80 »$, et le bouton « soumettre ». Je peux modifier le montant et 


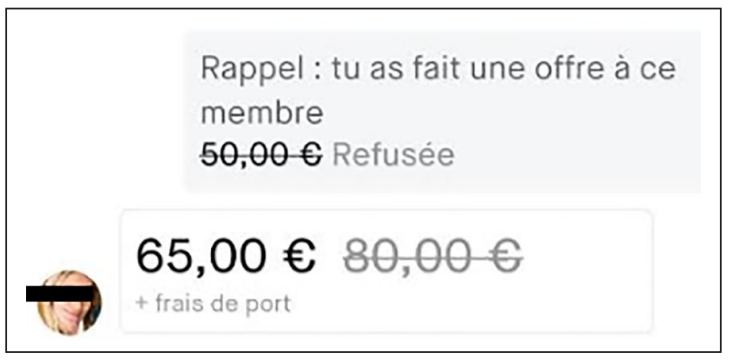

Illustration 4. Capture d'écran sur l'application Vinted.

mettre « 50 » par exemple, sans aucune explication à la vendeuse. La vendeuse propose en retour « 65 » sans aucune autre explication.

Cette affordance permet d'entrer en contact avec l'acheteuse sans investir l'énergie et le temps nécessaire à la construction d'une relation marchande significative, ce qui témoigne d'une aliénation par rapport aux autres (Rosa, 2012).

Les affordances ont permis également une accélération dans le domaine du transport. Parmi ces affordances se trouve le choix entre plusieurs options d'envoi au moment de la commande. En 2013, les utilisatrices devaient se rendre à la poste pour envoyer leur paquet ou passer par la remise en main propre. Djamila (39 ans) relate tout le temps que nécessitait la logistique des envois : «Je peux passer $3 \mathrm{~h}$ le dimanche, mais le plus dur c'est d'emballer, d'encaisser les chèques, d'aller à la poste. » Les livraisons deviennent par la suite plus rapides et moins contraignantes grâce à la possibilité offerte par les plateformes de passer notamment par Mondial Relay, son réseau dense de points de contact aux larges plages horaires, et son bordereau à imprimer chez soi. Julie (22 ans) explique : " Mondial Relay, moi j'adore, quand je fais une vente, j'ai juste à imprimer et à déposer au coin de la rue. C'est ouvert tard, 19h - 20h. La Poste, le matin 8 h30 ou 17h, quand tu travailles c'est moins évident ». Sandra (48 ans) partage ce point de vue : «Avec Mondial Relay, c'est vrai, c'est top! Tu déposes, tu imprimes de chez toi, tu ne perds pas de temps. Cela va plus vite qu'avant, largement plus vite. \C $\mathrm{Ce}$ mode d'acheminement intermédié réduit le temps entre le moment de l'achat et celui de la livraison. Il se distingue de la remise en main propre qui fait de la rencontre entre acheteur et vendeur une expérience sociale potentiellement riche et agréable (Garcia-Bardidia, 2014). Outre la diversification des options d'envoi, les trois plateformes étudiées délivrent des informations sur la livraison en estimant la durée de l'acheminement au moment de la commande, et en donnant la possibilité de suivre le colis.

Le corpus montre également que le temps consacré au paiement est compressé grâce à la création de nouvelles affordances facilitant la procédure d'achat. Les trois plateformes digitales étudiées offrent le choix entre plusieurs options de paiement, dont la carte bancaire, PayPal et le portefeuille virtuel qu'elles gèrent elles-mêmes. Pour présenter son portefeuille, la plateforme Vestiaire Collective insiste sur la rapidité des transferts d'argent qu'il permet : " Le portefeuille vous permet de recevoir le montant de vos ventes, dès qu'elles ont été réalisées, sans délais supplémentaires. Vous pouvez y placer votre argent pour régler vos futures acquisitions du Vestiaire Collective, ou le transférer vers votre compte bancaire. ». En plus d'accélérer les transferts d'argent, le portefeuille virtuel permet aux utilisatrices d'acheter toujours plus rapidement et de " craquer » facilement. A propos de l'argent qu'elle gagne de ses ventes, Julie (22 ans) explique : «Bon à l'origine, je devais le verser sur mon compte bancaire... T'es pris au jeu de Vinted, t'achètes. Je laisse maintenant sur Vinted.. . Aujourd'hui, je réutilise. Je laisse dans Vinted, de toute façon je vais acheter. » Le portefeuille virtuel facilite le rebond de consommation suscité par les gains des ventes (Denegri-Knott et Molesworth, 2009). Pour celles qui utilisent toujours leur carte bancaire, l'enregistrement des coordonnées bancaires permet d'effectuer des achats très rapidement. Toutes les utilisatrices interviewées évoquent la rapidité du paiement sur les plateformes digitales, dont Nina (20 ans) : "Je clique sur 'acheter', ensuite il faut entrer tes coordonnées bancaires, si tu ne les as pas déjà mis. Moi par exemple, elles sont déjà mises sur l'application donc j'appuie juste sur 'acheter' et je valide l'achat et voilà en 30 secondes, même pas, c'est fait!». Cette maitrise des données constitue un facteur clé du succès des plateformes (Filser et al., 2019). 


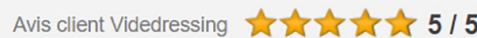

Aucun souci dans le parcours. C'était fluide et rapide. Vêtement livré rapidement en point relai. Rien à dire.

le 25/07/2019 par Anonymous. suite à une expérience du 23/06/2019

Illustration 5. Capture d'écran sur la plateforme Vide Dressing.

L'accélération technique multi-domaines encourage une concurrence par la rapidité organisée autour de la découverte permanente et de la maitrise des nouvelles affordances permettant d'aller plus vite. D'ailleurs, Vinted propose même une alerte pour être informée des nouvelles fonctionnalités de la plateforme. Connaître et utiliser ces affordances offre un avantage compétitif. En effet, les utilisatrices averties sont mieux équipées que les autres pour mener leurs activités marchandes. $\mathrm{Au}$ contraire, celles qui ne maitrisent pas les affordances sont plus lentes, ce qui peut générer le mécontentement d'utilisatrices frustrées par la lenteur et la perte de ventes. Par exemple, dans le cas des transports, ne pas opter pour l'option de livraison la plus rapide peut créer une insatisfaction chez des acheteuses habituées à recevoir très vite leurs articles. Nina (20 ans) évoque d'ailleurs une vente pour laquelle elle a reçu une mauvaise évaluation à cause d'un délai d'envoi trop long au goût de l'acheteuse : "J'ai été mal notée parce que je n'ai pas envoyé le colis assez vite. Elle, elle n'avait pas envie d'attendre. " Les observations vont dans ce sens, et attestent que la rapidité des expéditions est valorisée (Illustration 5). Ainsi, maitriser les dernières fonctionnalités est un moyen d'être plus compétitive, et d'éviter de devenir hors-jeu.

L'analyse du corpus de données montre que les affordances des plateformes étudiées favorisent une concurrence par la rapidité entre les pratiquantes de vide-dressing en ligne. Cette concurrence par la rapidité se manifeste par trois injonctions : être la plus réactive, être la plus à la pointe, et être la plus avertie. La Figure 1 synthétise les résultats présentés dans cette section. Comme nous l'avons indiqué précédemment, nous avons présenté séparément les trois formes de concurrence par la rapidité par souci de clarté. Néanmoins celles-ci sont empiriquement intriquées. Par exemple, les articles nouvellement mis en ligne apparaissent à la fois dans les sections « nouveautés 》 des plateformes (affordance « marqueur de nouveauté ») et dans les alertes reçues par les utilisateurs (affordance « notification »), les secondes permettant systématiquement d'accéder aux premières à l'aide d'un lien. Par conséquent, l'accélération du rythme de vie induite par les notifications va de pair avec l'accélération du renouvellement des objets possédés induite par les marqueurs de nouveauté, et contribuent ensemble à l'assiduité des utilisatrices. Être la plus à la pointe implique d'être la plus réactive. De même, le temps de réponse des utilisateurs (affordance « marqueur temporel ») est compressé grâce aux modalités de mise en contact des plateformes proposant des messages pré-écrits voire dispensant de la rédaction de message (affordances " mise en contact» et « contenu scripté »). Par conséquent, l'accélération technique induite par les modalités de communication est liée à l'accélération du rythme de vie induite par les marqueurs temporels. Être la plus avertie permet d'être la plus à la pointe. Enfin, les plateformes expliquent aux utilisatrices comment utiliser les différents modes d'acheminement des colis (affordance «plusieurs options d'envoi ») dans leurs centres d'aide (affordance " tutoriels et rubriques conseils »). Par conséquent, l'accélération technique dans le domaine du transport est reliée à l'accélération du renouvellement des objets possédés induite par les conseils des plateformes. Être la plus avertie permet d'être la plus à la pointe. Les différentes formes d'accélération et manifestations de concurrence par la rapidité sont donc imbriquées les unes dans les autres.

\section{Discussion}

Après avoir présenté nos principaux résultats, nous les interprétons maintenant à la lumière de la littérature existante, en montrant que nous y apportons trois contributions. Notre première contribution théorique consiste à montrer les conséquences de l'accélération induite par les plateformes sur les relations entre utilisateurs. Ce faisant, nous prolongeons le travail pionnier de Denegri-Knott (2011) dans lequel les conséquences de l'accélération sont explorées à l'échelle de la 


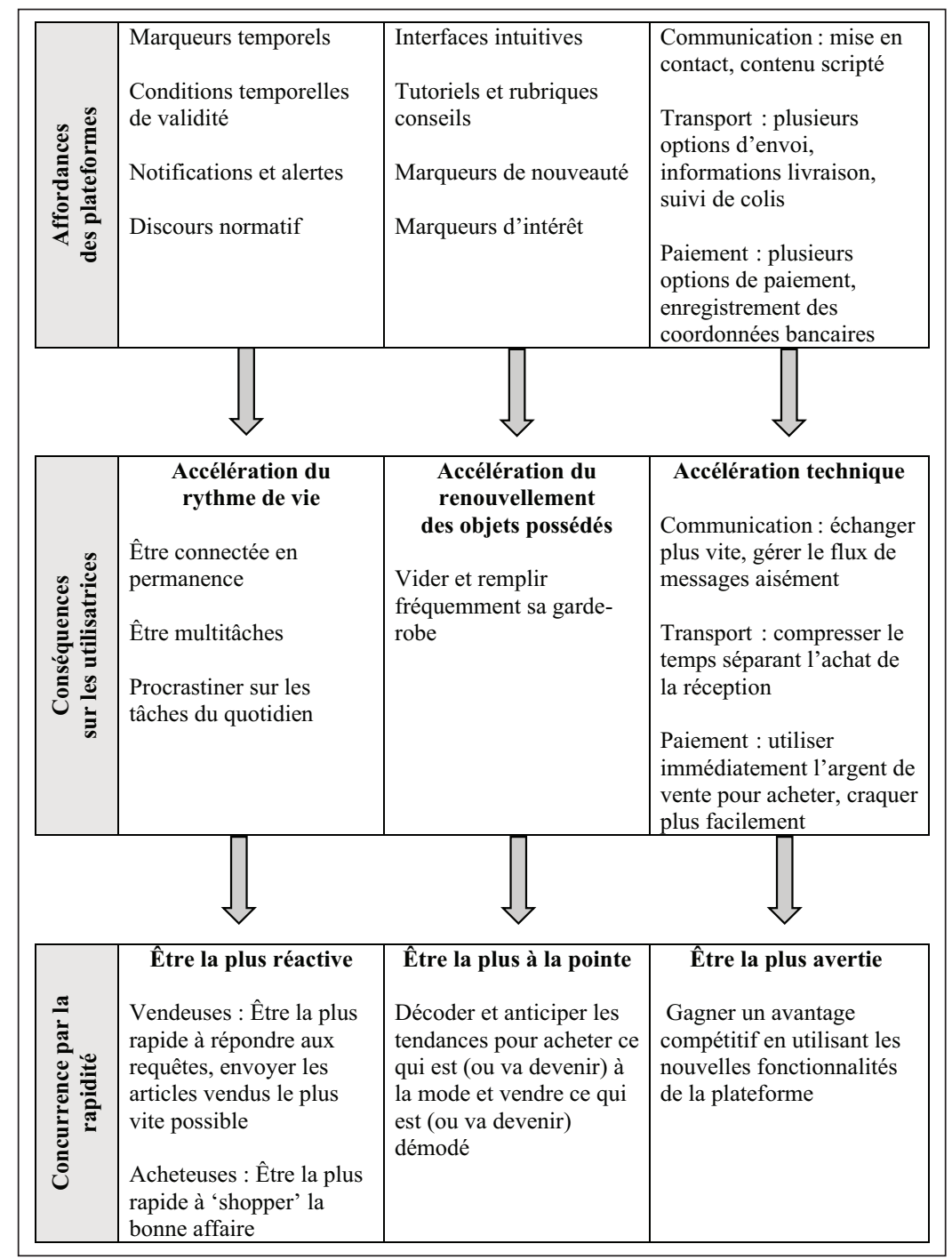

Figure I. Synthèse des résultats : Affordances, effets sur les utilisatrices et concurrence par la rapidité.

relation utilisateur-objet désiré. En élargissant la focale, nous montrons que l'accélération suscitée par les plateformes encourage des rapports concurrentiels entre utilisateurs. Dès lors, nos résultats contrastent avec les travaux ayant souligné la capacité des plateformes à créer des liens sociaux communaux entre utilisateurs. En effet, des recherches ont mis en lumière la formation de relations d'entraide entre demandeurs ou entre offreurs. Par exemple, Barraud de Lagerie et Sigalo Santos (2018) montrent comment une plateforme devient un espace chaleureux de sociabilité où les utilisateurs se soutiennent en cas de difficulté. Quant aux chauffeurs Uber, ils partagent leurs astuces de façon informelle pour s'entraider et protester contre un ennemi commun : la plateforme qui contraint drastiquement leur activité (Möhlman et Zalmanson 2017). Des liens sociaux se forment aussi entre offreurs et demandeurs. Les transactions répétées génèrent une confiance mutuelle qui incite les individus à ne pas respecter à la lettre toutes les prescriptions contraignantes imposées par la plateforme digitale (Bailly et Boudot-Antoine, 2018). Même lorsque les interlocuteurs font affaire 
pour la première fois, leur face-à-face peut représenter une expérience sociale agréable, notamment lorsque le vendeur reçoit l'acheteur chez lui et fait preuve d'hospitalité (GarciaBardidia, 2014). L'affabilité peut toutefois se transformer en injonction, comme c'est le cas des hôtes Airbnb qui doivent se montrer conviviaux avec les voyageurs (Jacquet, 2015), ou des chauffeurs de VTC qui doivent fournir un travail émotionnel vis-à-vis des passagers afin d'obtenir de bonnes évaluations (Rogers, 2017 ; Rosenblat et Stark, 2016). Néanmoins, toutes les plateformes n'encouragent pas à créer du lien social. Certaines plateformes sont caractérisées par un faible niveau de consocialité, au sens où elles offrent peu d'opportunités de communiquer, d'interagir, et donc de générer des bénéfices sociaux (Perren et Kozinets 2018). C'est par exemple le cas de Zipcar où les utilisateurs ne cherchent ni à se connecter les uns aux autres, ni à se rencontrer pour socialiser (Bardhi et Eckhardt, 2012). Dans la littérature, la question des relations sociales nourries par les plateformes a donc été appréhendée sous l'angle de leur intensité ou de leur spontanéité, mais non de leur nature : qu'elles soient nombreuses ou rares, spontanées ou travaillées, les relations sociales sont connotées positivement car elles sont de l'ordre de l'entraide et de la convivialité. Or, notre recherche montre que les relations sociales entre utilisateurs peuvent être de nature différente lorsqu'elles mettent en jeu des activités marchandes accélérées, et devenir concurrentielles. Ainsi, étudier les relations entre utilisateurs à l'aune de l'accélération permet de montrer la diversité des rapports sociaux encouragés par les plateformes digitales.

Notre deuxième contribution théorique revient à considérer la concurrence par la rapidité comme un moteur de l'intensité des activités marchandes sur les plateformes digitales. La littérature a déjà mis en lumière plusieurs raisons expliquant l'intensité des échanges sur les plateformes. Une première raison tient aux nombreuses opportunités d'ordre économique qui résonnent avec les motivations utilitaristes de la majorité des utilisateurs (Padmavathy et al., 2019). En effet, les plateformes permettent aux individus ayant un pouvoir d'achat limité de consommer, offrent de nombreux « bons plans » aux consommateurs malins, et génèrent un revenu complémentaire utilisé pour consommer plus
(Benavent, 2016 ; Peugeot et al., 2015 ; Robert et al., 2014). Une deuxième raison tient à la façon dont les utilisateurs perçoivent les plateformes digitales. Les utilisateurs succombent facilement à la tentation consumériste car ils voient les plateformes comme soutenables et produisant moins de déchets (Parguel et al, 2017). Une troisième raison tient à l'accélération du cycle de désir chez les utilisateurs (Denegri-Knott 2011). Les plateformes initient fréquemment de nouveaux cycles de désir en signalant en permanence de nouvelles options à acquérir, et permettent d'actualiser aisément des plaisirs immédiats (DenegriKnott, 2011 ; Moati, 2016). Nous complétons ces travaux en proposant une nouvelle raison - qui n'exclut pas les précédentes - expliquant l'intensité des activités marchandes et l'hyperconsommation sur les plateformes digitales : la mise en concurrence par la rapidité des utilisateurs. Les utilisateurs sont en concurrence pour être le plus réactif possible afin de ne rater aucune opportunité. Ils sont également en concurrence pour mettre à jour leurs possessions au plus vite, et ainsi être les plus à la pointe. Ils sont enfin en concurrence pour découvrir les dernières fonctionnalités des plateformes et gagner ainsi un avantage compétitif par rapport aux autres utilisateurs. Cette mise en concurrence permanente par la rapidité retient les utilisateurs dans une activité marchande intense, et cela même si le désir de consommer peut s'éroder à cause de cycles rapides trop souvent répétés (Denegri-Knott, 2011). Suivre le tempo dicté par les plateformes devient une fin en soi, le désir portant sur l'acquisition et la vente rapides d'objets, et non sur leur utilisation prolongée et signifiante (Rosa 2017). Dès lors, les plateformes digitales constituent des systèmes marchands " auto-propulsés ». "Auto » renvoie au fait que ce sont leurs propres affordances qui génèrent la mise en concurrence ; " propulsé » souligne qu'elles mettent en mouvement des utilisateurs aliénés animés par l'envie de ne pas se faire doubler. Loin d'être un modèle alternatif à l'économie marchande capitaliste, les plateformes digitales en sont une extension (Chabault, 2020 ; Juge et al., 2019).

Notre troisième contribution enrichit la littérature sur l'accélération. Rosa (2012) indique que l'accélération sociale suscite une aliénation des individus existant sous cinq formes : l'aliénation par rapport aux choses, l'aliénation par rapport aux actions, l'aliénation par rapport aux autres, 
l'aliénation par rapport au temps et l'aliénation par rapport à l'espace. Les résultats de notre recherche font apparaitre des manifestations des trois premières formes d'aliénation. Néanmoins, nos résultats invitent à discuter les deux dernières formes d'aliénation. Nous avons, en effet constaté que les utilisateurs sont connectés en permanence aux plateformes digitales, et cela quelle que soit leur activité en cours. En d'autres termes, les activités marchandes constituent le fil conducteur de la journée, et créent un lien entre les différents épisodes (travail, transport, domicile). Ceci nuance la notion d'aliénation par rapport au temps définie par Rosa (2012) comme la propension à cumuler une série de courtes expériences déconnectées entre elles. Il semble qu'au contraire, l'accélération des activités sur les plateformes digitales génère une continuité entre des expériences fractionnées et indépendantes les unes des autres. De plus, plusieurs affordances identifiées dans les données permettent aux utilisateurs de s'approprier l'espace digital qu'est la plateforme : les alertes reçues sont fonction des requêtes personnalisées, le contenu de la liste de souhaits dépend des articles qu'ils y ajoutent, et l'historique des transactions, les coordonnées bancaires et l'adresse de livraison sont propres à chaque utilisateur. En d'autres termes, les affordances sont communes à tous les utilisateurs tout en permettant à chacun d'eux d'investir personnellement la plateforme et de la transformer en un espace personnel singulier. Ceci nuance la notion d'aliénation par rapport à l'espace définie par Rosa (2012) comme un désengagement, un désinvestissement de l'espace physique ou matériel dans lequel on évolue du fait d'une mobilité accrue. Il semble qu'au contraire, plus les utilisateurs arpentent les plateformes, plus ils les investissent et se les approprient.

\section{Conclusion}

\section{Recommandations pratiques}

Notre recherche permet d'apporter des contributions pratiques ayant une portée sociétale. Nous proposons des pistes concrètes pour dévoiler aux utilisateurs l'ampleur de leurs activités marchandes accélérées et de leur mise en concurrence par la rapidité. Certes, aucun des utilisateurs interrogés ne s'est plaint ni n'a évoqué de souffrance liée à l'injonction à la rapidité. Néanmoins, " le propre de l'aliénation est de maintenir ceux qui en sont victimes dans la méconnaissance de leur condition » (Keucheyan, 2019 : 45). Nous n'affirmons pas que la prise de conscience va abolir l'aliénation (Keucheyan, 2019), ni supplanter le plaisir de l'activité marchande. Comme l'indiquent Béji-Bécheur et ÖzçağlarToulouse (2014), espérer que les consommateurs adoptent un comportement responsable et durable serait un vœu pieu. L'enjeu est simplement de permettre aux utilisateurs d'agir de façon éclairée. Pour cela, nous invitons les pouvoirs publics à réguler les plateformes digitales afin qu'elles modifient leurs affordances.

Pour que les utilisateurs prennent conscience de la façon dont les plateformes digitales les mettent en concurrence pour être le plus réactif, nous suggérons de créer une nouvelle affordance indiquant le temps cumulé passé sur l'application. Ce repère temporel donnerait aux utilisateurs une mesure objective de l'ampleur de leur activité. Ceci pourrait les aider à décrocher des plateformes, et générer ainsi une décélération technologique (Husemann et Eckhardt 2018). Passer moins de temps connecté sur les plateformes permettrait par ailleurs de diminuer le temps passé devant les écrans, et de baisser la consommation énergétique des utilisateurs. Celle-ci est le fait de centres de données particulièrement énergivores, de la navigation sur des pages contenant de nombreuses photos, du stockage des données, et de la conservation des messages envoyés et reçus dans le cadre des transactions ${ }^{9,10}$.

Pour que les utilisateurs sortent de l'accélération du renouvellement des objets possédés, nous suggérons de modifier une affordance existante. A ce jour, tout article ajouté à la liste de souhaits et acheté par un autre utilisateur plus rapide continue d'apparaitre dans la liste de souhaits, avec la mention «vendu ». De ce fait, les utilisateurs ne peuvent que constater les opportunités manquées en raison de leur lenteur ou de leur indécision. Nous suggérons de supprimer les « occasions ratées 》 frustrantes de la liste de souhaits car elles sont susceptibles d'encourager les utilisateurs à acheter des articles sans forcément prendre le temps de la réflexion, uniquement par peur qu'un autre utilisateur soit plus rapide qu'eux. 
Pour que les utilisateurs prennent conscience de la façon dont l'accélération technique dans le domaine du paiement contribue à intensifier leurs activités marchandes, nous suggérons de créer une nouvelle affordance. A ce jour, les utilisateurs voient la cagnotte dont ils disposent grâce à leurs ventes, et le prix de chaque item déjà acheté. Ceci leur donne l'impression d'avoir gagné beaucoup d'argent grâce à leurs ventes. Pour contrebalancer cet effet, nous suggérons d'ajouter une affordance qui ferait la somme de toutes les dépenses cumulées et indiquerait aux utilisateurs le montant total dépensé depuis la création de leur profil. Comme les items disponibles sur les plateformes digitales sont moins chers que sur le marché du neuf, les utilisateurs n'ont pas nécessairement conscience de la somme totale d'argent qu'ils dépensent. Cette nouvelle affordance montrerait aux utilisateurs qu'ils gagnent certes un complément de revenus grâce aux plateformes, mais qu'ils dépensent tout autant - si ce n'est davantage.

\section{Limites et programme de recherche}

En dépit de la richesse de notre corpus et de nos contributions, notre travail présente trois limites principales. La première est la composition de notre échantillon, lequel est exclusivement féminin, et majoritairement jeune pour ce qui concerne les entretiens. Si notre choix d'interroger uniquement des utilisatrices est justifié par la présence mineure des hommes sur les plateformes de vide-dressing ${ }^{11}$, il n'empêche qu'il invisibilise la faible proportion d'hommes actifs sur ces plateformes. Une voie de recherche consisterait donc à explorer le comportement des hommes pratiquant le videdressing en ligne afin de brosser un portrait complet et inclusif de cette pratique marchande. Toujours dans la perspective d'avoir une approche plus inclusive de la pratique, il conviendrait aussi d'interroger des femmes plus âgées, certes minoritaires sur les plateformes digitales mais dont le rapport aux nouvelles technologies serait moins fluide d'après Ritzer et Miles (2019). La seconde limite de notre étude est la restriction à un seul marché. Il serait pertinent de compléter notre analyse du cas du marché du vêtement d'occasion en explorant des plateformes opérant dans d'autres secteurs comme la rencontre amoureuse, le transport et l'hébergement. L'étude d'autres plateformes permettrait d'identifier peut-être d'autres manifestations de la concurrence par la rapidité et ainsi de renforcer le mécanisme mis en lumière dans cet article. La troisième limite est relative à l'évolution permanente des fonctionnalités des plateformes digitales. Il est possible que de nouvelles affordances soient apparues ou aient disparues une fois notre collecte de données terminée. Une voie de recherche consisterait à continuer à étudier l'évolution des affordances des plateformes digitales au cours du temps afin de comprendre comment elles contribuent au renforcement ou au contraire à la diminution de la concurrence par la rapidité.

Une première voie de recherche consisterait à répondre à la question suivante : Dans quelle mesure la covid-19 transforme-t-elle la pratique du videdressing en ligne ? Les motivations économiques étant prépondérantes dans l'achat d'occasion (Padmavathy et al., 2019), il est possible que la crise économique $^{12}$ génère un surcroît d'utilisateurs sur les plateformes et accroisse ainsi la concurrence par la rapidité. Le nombre de concurrents augmentant, il se pourrait qu'il faille alors aller encore plus vite pour ne pas être hors-jeu. Le confinement a par ailleurs incité certains Français à remettre en question les fondements de leur mode de vie et de la société $^{13}$. Une vie moins rapide, plus simple et plus écologique est apparue comme un scénario possible du 《 monde d'après $\|^{14}$. Il se pourrait que certains Français, soucieux de sortir du consumérisme et de décélérer, se dirigent vers les plateformes de vide-dressing. Il serait alors intéressant d'étudier comment ces nouveaux venus réagissent face à une concurrence par la rapidité inattendue sur ce type de plateforme prônant les arguments communautaires de l'économie collaborative, comment utilisateurs rapides et utilisateurs en quête de lenteur coexistent sur les plateformes, et comment une telle cohabitation peut générer des frictions temporelles.

Une seconde voie de recherche consisterait à répondre à la question suivante : quels sont les mécanismes conduisant à l'exclusion ou l'autoexclusion des utilisateurs de plateformes digitales de vide-dressing? Il est possible que certains vendeurs ne parviennent pas à suivre le rythme, et que leur lenteur soit sanctionnée dans les évaluations. Ces évaluations pourraient susciter la défiance des 
acheteurs, diminuer petit à petit le nombre de transactions conclues, et repousser les utilisateurs aux marges de la plateforme jusqu'à leur exclusion de fait. De même, des acheteurs pourraient aussi avoir des difficultés à suivre le rythme. Ceux-ci se feraient doubler systématiquement par d'autres acheteurs plus rapides, ce qui diminuerait leur nombre de transactions, et les exclurait de facto de la plateforme. D'autres utilisateurs pourraient quant à eux prendre conscience de la concurrence par la rapidité qui pèse sur eux, et ne plus pouvoir ou vouloir supporter le rythme imposé par les plateformes. Ces utilisateurs pourraient refuser les règles du jeu et abandonner d'eux-mêmes la pratique marchande. Il conviendrait de se pencher sur ces cas d'exclusion de fait et d'auto-exclusion et ainsi de mieux comprendre les «perdants » de la malnommée « économie collaborative ».

\section{ORCID iD}

Isabelle Collin-Lachaud (iD https://orcid.org/0000-0002 $-4783-6652$

\section{Notes}

1. https://www.un.org/fr/sections/issues-depth/ climate-change/index.html

2. https://fr.fashionnetwork.com/news/Quatrefrancais-sur-dix-sont-des-convertis-a-la-secondemain, 1142798.html

3. www.lemonde.fr/economie/article/2019/02/12/ la-france-meilleure-cliente-de-vinted_5422432 3234.html

4. https://www.lsa-conso.fr/vinted-le-roi-de-laseconde-main-au-volume-d-affaires-d-1-3-milliardd-euros,330371

5. www.lemonde.fr/economie/article/2019/02/12/ la-france-meilleure-cliente-de-vinted_5422432_ 3234.html

6. https://www.1sa-conso.fr/vinted-le-roi-de-laseconde-main-au-volume-d-affaires-d-1-3-milliardd-euros,330371

7. $10 \%$ seulement des utilisateurs de Vinted sont des hommes. Source : La Quotidienne du 24 janvier 2020 (France 5).

8. https://www.bfmtv.com/economie/les-chiffresfous-de-la-friperie-numerique-vinted-en-france1632560.html

9. https://www.greenpeace.org/usa/global-warming/ click-clean/

10. https://total.direct-energie.com/particuliers/parlons -energie/dossiers-energie/comprendre-le-marche- de-1-energie/la-consommation-energetique-de-1 -internet-mondial

11. $10 \%$ des utilisateurs de Vinted sont des hommes (Source : La Quotidienne du 24 janvier 2020 France 5).

12. https://www.lemonde.fr/economie/article/2020/ 04/10/coronavirus-quels-scenarios-pour-la-sortiede-crise-economique 6036168_3234.html

13. https:/www.lemonde.fr/societe/article/2020/05/10/ tout-plaquer-pour-une-vie-plus-simple-le-confine ment-declic-d-un-changement-de-vie_6039202_ 3224.html

14. https://www.lemonde.fr/idees/article/2020/04/13/ cyril-dion-la-crise-du-covid-19-peut-nous-aider-aconstruire-le-monde-d-apres_6036417_3232.html

\section{Références bibliographiques}

Akrich M et Latour B (1992) A summary of a convenient vocabulary for the semiotics of human and nonhuman assemblies. In: Bijker W et Law J (éds) Shaping Technology/Building Society: Studies in Sociotechnical Change. Cambridge MA: MIT Press, 259-264.

Allard-Poesi F (2015) Des méthodes qualitatives dans la recherche en management : Voies principales, tournants et chemins de traverse. In: Moriceau J-L et Soparnot R (éds) Pratiques des méthodes qualitatives. Paris: EMS.

Arnould EJ et Price LL (1993) River magic: extraordinary experience and the extended service encounter. Journal of Consumer Research 20(1): 24-45.

Arsel Z (2017) Asking questions with reflexive focus: a tutorial on designing and conducting interviews. Journal of Consumer Research 44(4): 939-948.

Assouly O (2010) Usage, design et mode. Disponible en ligne: https://www.ifmparis.fr/fr/recherche-academique/usages-design-et-mode [consulté le 10 février 2021]..

Badot O, Carrier C, Cova B, Desjeux D et Filser M (2009) L'ethnomarketing : un élargissement de la recherche en comportement du consommateur à l'ethnologie. Recherche et Applications en Marketing 24(1): 93-111.

Bailly A et Boudot-Antoine F (2018) Renforcement et transgression du cadre de l'intermédiation numérique. Réseaux (6): 121-148.

Bardhi F et Eckhardt GM (2012) Access-based consumption: the case of car sharing. Journal of Consumer Research 39(4): 881-898.

Bardini T (1996) Changement et réseaux socio-techniques : de l'inscription à l'affordance. Réseaux, 14(76): 125-155.

Barraud de Lagerie PB et Santos LS (2018) Et pour quelques euros de plus. Réseaux (6): 51-84. 
Beauvisage T, Beuscart JS et Mellet K (2018) Numérique et travail à-côté. Enquête exploratoire sur les travailleurs de l'économie collaborative. Sociologie $d u$ travail 60(2). Disponible en ligne: http://journals. openedition.org/sdt/1984 [consulté le 9 février 2021].

Béji-Bécheur A et Özçağlar-Toulouse N (2014) Institutionnalisation du développement durable et émergence d'un marketing durable. Recherche et Applications en Marketing. 29(3): 3-9.

Belk RW, Ger G et Askegaard S (2003) The fire of desire: a multisited inquiry into consumer passion. Journal of Consumer Research 30(3): 326-351.

Belk R (2014) You are what you can access: sharing and collaborative consumption online. Journal of Business Research 67(8): 1595-1600.

Benavent C (2016) Plateformes. Sites collaboratifs, marketplaces, réseaux sociaux... Comment ils influencent nos choix. Limoges: FYP éditions.

Bernard Y (2004) La netnographie : une nouvelle méthode d'enquête qualitative basée sur les communautés virtuelles de consommation. Décisions Marketing 36(octobre-décembre): 49-62.

Beuscart JS et Flichy P (2018) Plateformes numériques. Réseaux (6): 9-22.

Botsman R et Rogers R (2010) What's Mine is Yours. The Rise of Collaborative Consumption. New York: Harper Collins.

Bourdieu P (1981) Décrire et prescrire. Actes de la recherche en sciences sociales 38(1): 69-73.

Callon M, Méadel C et Rabeharisoa V (2000) L'économie des qualités. Politix. Revue des sciences sociales $d u$ politique 13(52): 211-239.

Callon M et Muniesa F (2003) Les marchés économiques comme dispositifs collectifs de calcul. Réseaux (6): $189-233$.

Chabault V (2020) Du livre-déchet au livre vendu. L'écologisation du marché du livre d'occasion. Ecologie et politique 60(1): 91-104.

Chen TD et Kockelman KM (2016) Carsharing's lifecycle impacts on energy use and greenhouse gas emissions. Transportation Research Part D: Transport and Environment 47: 276-284.

Decrop A (2017) La consommation collaborative : Enjeux et défis de la nouvelle société du partage. Bruxelles: De Boeck Supérieur.

Denegri-Knott J (2011) "Have it now!": ebBay and the acceleration of consumer desire. European Advances in Consumer Research 9: 373-379.

Denegri-Knott J et Molesworth M (2009) 'I'll sell this and I'll buy them that': eBay and the management of possessions as stock. Journal of Consumer Behaviour: An International Research Review 8(6): 305-315.
Denegri-Knott J et Molesworth M (2013) Redistributed consumer desire in digital virtual worlds of consumption. Journal of Marketing Management 29(13-14): 1561-1579.

Dumez H (2013) Méthodologie de la recherche qualitative; les 10 questions clés de la démarche compréhensive. Paris: Vuibert.

Ertz M, Durif F, Lecompte A et Boivin C (2018) Does "sharing" mean "socially responsible consuming"? Exploration of the relationship between collaborative consumption and socially responsible consumption. Journal of Consumer Marketing 35(4): 392-402.

Filser M, Henríquez T et Frisou J (2019) Plateforme contre canal. Quel cadre théorique pour analyser la distribution ? Revue française de gestion 283(6): 93-107.

Garcia-Bardidia R (2014) Se débarrasser d'objets sur leboncoin. fr. Une pratique entre don et marché ? Revue du MAUSS 44(2): 271-285.

Galluzzo A (2020) La fabrique du consommateur. Une histoire de la société marchande. Paris: La Découverte.

Gibson JJ (1977) The theory of affordances. In: Shaw RE et Bransford J (éds) Perceiving, Acting, and Knowing: Toward an Ecological Psychology. Hillsdale NJ: Lawrence Erlbaum Associates, 67-82.

Gourmelen A, Urien B, et Le et Gall-Ely M (2016) Le concept de pression temporelle ultime et sa mesure. Recherche et Applications en Marketing 31(4): 5-29.

Greeno J (1994): Gibson's affordances. Psychological Review 101(2): 336-342.

Groleau C (2003) L'observation. In: Giordano Y Conduire un projet de recherche. Une perspective qualitative. Paris: EMS.

Hamari J, Sjöklint M et Ukkonen A (2015) The sharing economy: why people participate in collaborative consumption. Journal of the Association for Information Science and Technology. 67(9): 2047-2059.

Husemann KC et Eckhardt GM (2018) Consumer deceleration. Journal of Consumer Research 45(6): 1142-1163.

Hutchby I (2001) Technologies, texts and affordances. Sociology 35(2): 441-456.

Jacquet E (2015) Le « prêt payant ». Les paradoxes de l'économie collaborative. Réseaux (2): 99-120.

Juge E, Collin-Lachaud I et Roux D (2019) Extension du domaine de l'entrepreneurialité dans la pratique du videdressing. Revue Française de Gestion 45(284): 31-49.

Kenney M et Zysman J (2016) The rise of the platform economy. Issues in Science and Technology 32(3): 61.

Keucheyan R (2019) Les besoins artificiels : Comment sortir du consumérisme. Paris: La Découverte.

Klein N (2019) Plan B pour la planète : Le New Deal vert. Paris: Actes Sud. 
Kopytoff I (1986) The cultural biography of things: commoditization as process. The Social Life of Things: Commodities in Cultural Perspective : 64-92.

Kozinets R, Patterson A et Ashman R (2017) Networks of desire: how technology increases our passion to consume. Journal of Consumer Research 43(5): 659-682.

Lallement J et Gourmelen A (2018) Le temps des consommateurs : état des recherches et perspectives. Recherche et Applications en Marketing 33(4): 98-131.

Latour B (2015) Face à Gaïa. Paris: La Découverte.

Leonardi PM (2011) When flexible routines meet flexible technologies: affordance, constraint, and the imbrication of human and material agencies. MIS Quarterly 35(1): 147-167.

Leonardi PM et Vaast E (2017) Social media and their affordances for organizing: a review and agenda for research. Academy of Management Annals 11(1): 150-188.

Light B, Burgess J et Duguay S (2018) The walkthrough method: an approach to the study of apps. New Media \& Society 20(3): 881-900.

Luckman S (2013) The aura of the analogue in a digital age: women's crafts, creative markets and homebased labour after Etsy. Cultural Studies Review 19(1): 249-70.

Maciel AF et Wallendorf M (2017) Taste engineering: an extended consumer model of cultural competence constitution. Journal of Consumer Research 43(5): 726-746.

Michaud-Trévinal A et Stenger T (2018) Pour un renouvellement du concept de situation : le cas de la situation de shopping en ligne à domicile. Recherche et Applications en Marketing 33(4): 27-49.

Miles MB et Huberman AM (1994) Qualitative Data Analysis. $2^{\text {éme }}$ edition. Thousand Oaks CA: SAGE.

Moati P (2016) Vers la fin de la grande distribution ? Revue française de socio-économie 16(1): 99-118.

Möhlmann M et Zalmanson L (2017) Hands on the wheel: navigating algorithmic management and Uber drivers. In: 'Autonomy', in Proceedings of the International Conference on Information Systems (ICIS). Seoul, South Korea 10-13.

Norman DA (1988) The Psychology of Everyday Things. New York: Basic Book.

Padmavathy C, Swapana M et Paul J (2019) Online second-hand shopping motivation-conceptualization, scale development, and validation. Journal of Retailing and Consumer Services : 19-32.

Parchoma G (2014) The contested ontology of affordances: implications for researching technological affordances for collaborative knowledge production. Computers in Human Behavior 37: 360-368.

Parguel B, Lunardo R et Benoit-Moreau F (2017) Sustainability of the sharing economy in question: when second-hand peer-to-peer platforms stimulate indulgent consumption. Technological Forecasting and Social Change 125: 48-57.

Parguel B, Monnot E, Reniou F et Benoit-Moreau F (2018) Les finalités sociétales dans la recherche francophone en marketing. Revue française de gestion (7): 11-31.

Perren R et Kozinets RV (2018) Lateral exchange markets: how social platforms operate in a networked economy. Journal of Marketing 82(1): 20-36.

Peugeot V, Beuscart JS, Pharabod AS et Trespeuch M (2015) Partager pour mieux consommer ? Esprit (7): $19-29$.

Piketty T (2013) Le Capital au XXIème siècle. Paris: Le Seuil.

Point S et Voynet-Fourboul CV (2006) Le codage à visée théorique. Recherche et Applications en Marketing 21(4): 61-78.

Ritzer G et Miles S(2019) The changing nature of consumption and the intensification of McDonaldization in the digital age. Journal of Consumer Culture 19(1): $3-20$.

Robert I, Binninger AS et Ourahmoune N (2014) La consommation collaborative, le versant encore équivoque de l'économie de la fonctionnalité. Développement durable et territoires. Economie, géographie, politique, droit, sociologie 5(1): 1-30.

Rogers B (2017) The social cost of Uber. University of Chicago Law Review Online 82(1): 85-102.

Rosa H (2012) Accélération. Une critique sociale du temps. Paris: La Découverte.

Rosa H (2017) Résonance. Une sociologie de la relation au monde. Paris: La Découverte.

Rosenblat A et Stark L (2016) Algorithmic labor and information asymmetries: a case study of Uber's drivers. International Journal of Communication 10. Disponible en ligne: http://dx.doi.org/10.2139/ ssrn.2686227 [consulté le 11 février 2021].

Royer I et Zarlowski P (2007) Le design de la recherche. In: Raymond-Alain Thiétart RA (éd.) Méthodes de recherche en Management. $3^{\text {ème }}$ édition, Paris: Dunod, 168-196.

Sandikci Ö et Ger G (2010) Veiling in style: how does a stigmatized practice become fashionable? Journal of Consumer Research 37(1): 15-36.

Spiggle S (1994) Analysis and interpretation of qualitative data in consumer research. Journal of Consumer Research 21(3): 491-503. 
Suthers DD (2006) Technology affordances for intersubjective meaning making: a research agenda for CSCL. International Journal of Computer-Supported Collaborative Learning 1(3): 315-337.
Zervas G, Proserpio D et Byers JW (2017) The rise of the sharing economy: estimating the impact of Airbnb on the hotel industry. Journal of Marketing Research 54(5): 687-705.

Annexe I. Synthèse chronologique de la collecte des données réalisée entre 2013 et 2020.

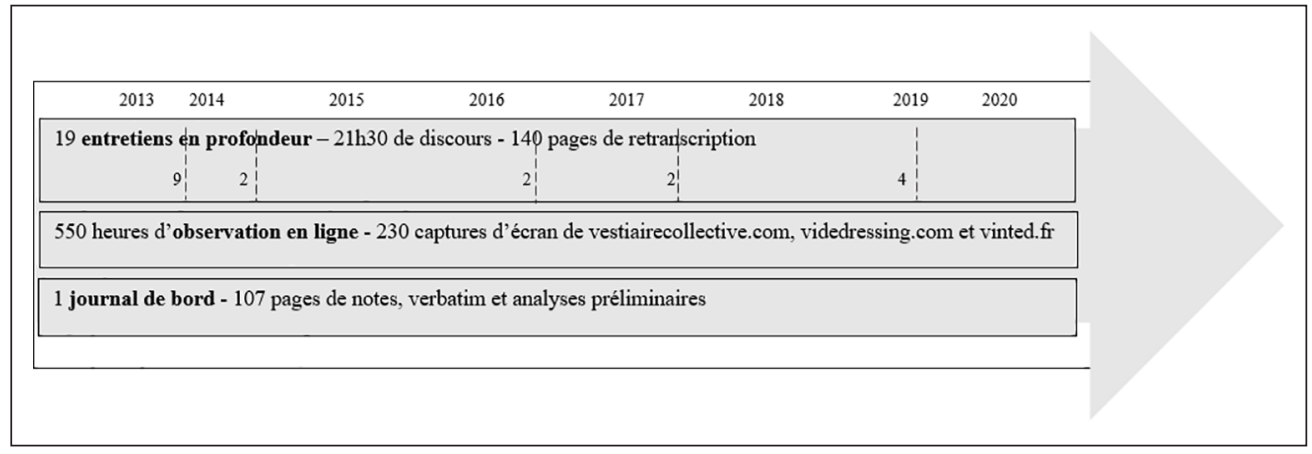

Annexe 2. Portraits des 19 pratiquantes interrogées entre mai 2013 et décembre 2019.

\begin{tabular}{|c|c|c|c|c|c|c|}
\hline $\begin{array}{l}\text { Pseudonyme choisi } \\
\text { par la répondante }\end{array}$ & $\begin{array}{l}\text { Date de } \\
\text { l'entretien }\end{array}$ & $\begin{array}{l}\text { Durée de } \\
\text { l'entretien }\end{array}$ & Age & $\begin{array}{l}\text { Occupation profes- } \\
\text { sionnelle }\end{array}$ & $\begin{array}{l}\text { Situation per- } \\
\text { sonnelle }\end{array}$ & Plateforme utilisée \\
\hline Amandine & $9 / 09 / 3013$ & $62 \mathrm{mn}$ & 23 ans & Assistante RH & $\begin{array}{l}\text { Célibataire } \\
\text { Sans enfant }\end{array}$ & Vinted \\
\hline Estelle & $11 / 09 / 2013$ & $67 \mathrm{mn}$ & 23 ans & Responsable qualité & $\begin{array}{l}\text { En couple } \\
\text { Sans enfant }\end{array}$ & $\begin{array}{l}\text { Vinted, Vestiaire } \\
\text { Collective }\end{array}$ \\
\hline Florence & $11 / 09 / 2013$ & $60 \mathrm{mn}$ & 43 ans & Infirmière & $\begin{array}{l}\text { En couple } \\
\text { I enfant }\end{array}$ & Vinted \\
\hline Clémence & $17 / 09 / 2013$ & $68 \mathrm{mn}$ & 22 ans & Infographiste & $\begin{array}{l}\text { En couple } \\
\text { Sans enfant }\end{array}$ & Vinted, Vide Dessing \\
\hline Shéhérazade & $17 / 09 / 2013$ & $72 \mathrm{mn}$ & 26 ans & Employé de banque & $\begin{array}{l}\text { Célibataire } \\
\text { Sans enfant }\end{array}$ & $\begin{array}{l}\text { Vinted, Vestiaire } \\
\text { Collective }\end{array}$ \\
\hline Ophélie & $19 / 09 / 2013$ & $63 \mathrm{mn}$ & 23 ans & Vendeuse de meuble & $\begin{array}{l}\text { En couple } \\
\text { Sans enfant }\end{array}$ & Vinted \\
\hline Stéphanie & $24 / 09 / 2013$ & $64 \mathrm{mn}$ & 38 ans & $\begin{array}{l}\text { Educatrice } \\
\text { spécialisée }\end{array}$ & $\begin{array}{l}\text { En couple } \\
\text { I enfant }\end{array}$ & Vinted \\
\hline Manon & $26 / 09 / 2013$ & $74 \mathrm{mn}$ & 25 ans & $\begin{array}{l}\text { Gestionnaire } \\
\text { d'approvisionnement }\end{array}$ & $\begin{array}{l}\text { En couple } \\
\text { Sans enfant }\end{array}$ & Vinted, Vide Dessing \\
\hline Djamila & $27 / 09 / 2013$ & $73 \mathrm{mn}$ & 39 ans & Enseignante & $\begin{array}{l}\text { En couple } \\
2 \text { enfants }\end{array}$ & Vinted, Vide Dessing \\
\hline Claire & $5 / 05 / 2014$ & $67 \mathrm{mn}$ & 20 ans & $\begin{array}{l}\text { Etudiante en } \\
\text { architecture }\end{array}$ & $\begin{array}{l}\text { Célibataire } \\
\text { Sans enfant }\end{array}$ & Vinted \\
\hline Léonie & $12 / 05 / 20 \mid 4$ & $86 \mathrm{mn}$ & 24 ans & Photographe & $\begin{array}{l}\text { Célibataire } \\
\text { Sans enfant }\end{array}$ & $\begin{array}{l}\text { Vinted, Vestiaire } \\
\text { Collective }\end{array}$ \\
\hline Jade & $21 / 09 / 2016$ & $71 \mathrm{mn}$ & 20 ans & Etudiante en lettre & $\begin{array}{l}\text { En couple } \\
\text { Sans enfant }\end{array}$ & Vinted, Vide Dessing \\
\hline Charlotte B & $10 / 10 / 2016$ & $92 \mathrm{mn}$ & 25 ans & Sans emploi & $\begin{array}{l}\text { En couple } \\
\text { Sans enfant }\end{array}$ & $\begin{array}{l}\text { Vinted, Vestiaire } \\
\text { Collective, } \\
\text { Vide Dessing }\end{array}$ \\
\hline
\end{tabular}


Annexe 2. (Suite)

\begin{tabular}{|c|c|c|c|c|c|c|}
\hline $\begin{array}{l}\text { Pseudonyme choisi } \\
\text { par la répondante }\end{array}$ & $\begin{array}{l}\text { Date de } \\
\text { l'entretien }\end{array}$ & $\begin{array}{l}\text { Durée de } \\
\text { l'entretien }\end{array}$ & Age & $\begin{array}{l}\text { Occupation profes- } \\
\text { sionnelle }\end{array}$ & $\begin{array}{l}\text { Situation per- } \\
\text { sonnelle }\end{array}$ & Plateforme utilisée \\
\hline Laëtitia & $09 / 12 / 2017$ & $57 \mathrm{mn}$ & 22 ans & Coach sportif & $\begin{array}{l}\text { En couple } \\
\text { Sans enfant }\end{array}$ & Vinted \\
\hline Inès & $18 / 12 / 2017$ & $70 \mathrm{mn}$ & 22 ans & Analyste web & $\begin{array}{l}\text { En couple } \\
\text { Sans enfant }\end{array}$ & Vinted, Vide Dessing \\
\hline Charlotte & $06 / 01 / 2019$ & $84 \mathrm{mn}$ & 28 ans & Sage-femme & $\begin{array}{l}\text { En couple } \\
\text { I enfant }\end{array}$ & Vinted \\
\hline Julie & $06 / 01 / 2019$ & $76 \mathrm{mn}$ & 22 ans & Secrétaire & $\begin{array}{l}\text { En couple } \\
\text { I enfant }\end{array}$ & Vinted \\
\hline Sandra & $23 / 12 / 2019$ & $68 \mathrm{mn}$ & 48 ans & Audio-prothésiste & $\begin{array}{l}\text { En couple } \\
2 \text { enfants }\end{array}$ & Vinted, Vide Dessing \\
\hline Nina & $28 / 12 / 2019$ & $82 \mathrm{mn}$ & 20 ans & Elève infirmière & $\begin{array}{l}\text { Célibataire } \\
\text { Sans enfant }\end{array}$ & Vinted \\
\hline
\end{tabular}

Annexe 3. Illustration du travail de triangulation des données collectées par les différentes méthodes (entretiens, observations en ligne, journal de bord).

\section{$>$ Entretien}

Jade (20 ans) souligne qu'elle achète le plus vite possible quand elle a une réponse rapide sur son offre de prix pour ne pas rater la bonne affaire: « je mets l'article dans mes favoris et dès que j'ai la réponse de la femme, bah je ne traine pas, sinon je me fais griller, y'a quelqu'un d'autre qui l'achète. » $>$ Observation sur application https://www.vinted.fr/app

Accélération du

rythme de vie

Glisse Vinted dans ta poche Télécharge l'application gratuite pour iOS ou Android et emporte Vinted partout avec tor! Fais pause déjeuner et échange en te baladant.
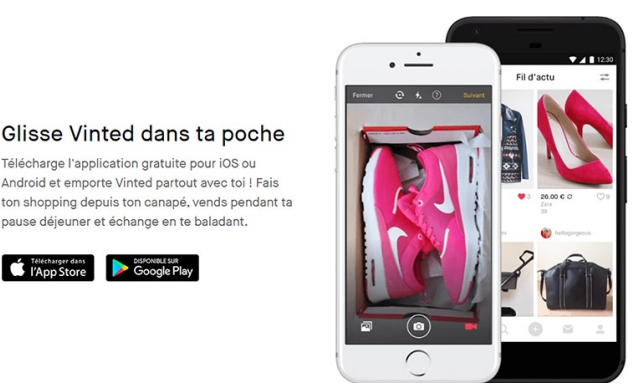

https://fr.VestiaireCollective.com/vendre-vetement-occasion/

Être la plus réactive

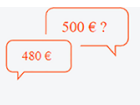

SOYEZ RÉACTIF

Les acheteurs peuvent effectuer des offres d'achat. Rester ouvert(e) à ces offres vous permettra de vendre encore plus rapidement. Vous avez également la possibilité de faire une offre aux acheteurs ayant commenté votre article.

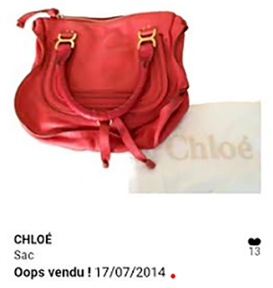

\section{Journal de bord}

Mars 2019, dans les transports en commun à une jeune fille sur l'application Vinted sur son smartphone

Observateur : « Je peux me permettre de vous demander, que regardez-vous sans arrêt sur votre Iphone?")

Réponse de l'observée : « Je vends des habits sur Vinted, je regarde s'il y a de l'activité pour répondre vite sinon les filles elles achètent autre chose. » 
Accélération du renouvellement des objets possédés

Être la plus à la pointe

\section{Entretien}

Laëtitia (22 ans) s'est inscrite facilement sur la plateforme Vinted " pour s'inscrire non rien de particulier, ça va vite, c'est comme les autres, en 2/2 c'est fait! » Estelle (23 ans) cherche à « recycler [son] armoire rapidement » et Nina (20 ans) peut « changer de vêtements plus souvent ».

Observation sur ordinateur https://www.VideDessing.com/

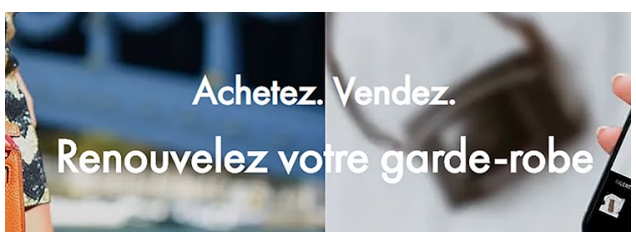

https://www.vinted.fr/how_it_works

Vendre, c'est simple
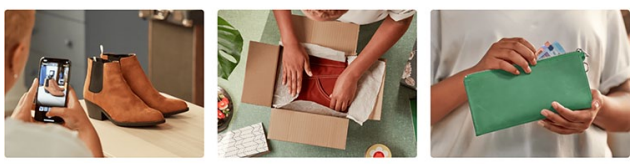

1. Mets en ligne gratuitement

2. Vends et envoie facilement

3. Jour de paie !

\|l y a o frais de vente, ce que tu

Télécharge l'appli gratuite Vinted.

Prends ton article en photo, décrile et fixe ton prix. Clique sur

"Ajouter" et c'est en ligne

Vendu ! Emballe ton article et

gagnes est à toi. Tu recerras

I'argent quand l'acheteur aure imprime le bordereau d'envol. Tu as Pargent quand Fachereur au 5 jours pour le déposer au point Relais proche de chez toi. En saveir plus

En savoir plus En sayoit dlus

\section{Journal de bord}

Mai 2014 lecture de blog se rapportant aux plateformes de vide-dressing en ligne https://vireedanslespace.wordpress.com/20 I4/05//8/\%E2\%96\%B2-jour-20-jevous-presente-mon-grand-amour-vinted-fr/

https://madame.lefigaro.fr/style/trois-sites-pour-gagner-de-largent-revendant-sesvetements-|009|4-913169

https://www.monsieurasgard.com/vinted-renouvelez-sans-vous-ruiner/ 
Accélération technique multidomaines

Être la plus avertie

\section{Entretien}

Manon (25 ans) : « Sur les sites de vide-dressing il faut que tu sois bien au courant de tout, enfin de toutes les possibilités comme pour les envois, les paiements, les retours, pleins de choses . . . tu n'as pas intérêt à déconner (rire) ». Sandra (48 ans) : « Avec Mondial Relay, c'est vrai, c'est top! Tu déposes, tu imprimes de chez toi, tu ne perds pas de temps. Cela va plus vite qu'avant, largement plus vite. ॥

\section{$>$ Observation sur ordinateur https://fr.VestiaireCollective.com/}

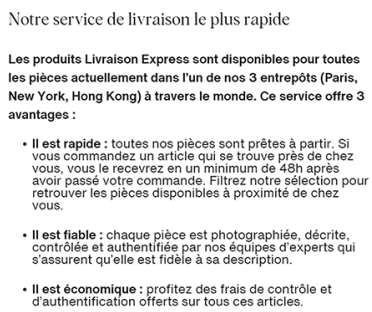$$
\text { Q Vendeur Recommandé } \quad 0 \bigcirc
$$

Vérifiez si votre pièce est éligible au service Livraison Express on ropérant lo symbole

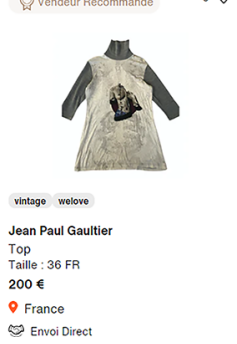

\section{Journal de bord}

Septembre 2018, lors d'une conversation entre amies Observateur : « Toi aussi tu vends tes fringues en ligne? !!!»

Réponse de l'observée : «Bah oui, tu ne savais pas? mais il faut être à la page, c'est Marion (sa fille) qui m'explique Paypal car moi . . .. au début je ne connaissais pas mais maintenant, je suis obligée, elles veulent toutes payer avec ça. Remarque ça va vite, c'est bien. »

Avril 2019, lors d'observation en ligne sur ordinateur Sur le site Vestiaire Collective, il y a une indication Vendeur Recommandé mais aussi une indication Envoi Direct, que signifie ces indications ??? je ne trouve pas de suite l'information, il me faut chercher sur le site. . . je ne trouve pas. Je fais donc une requête sur Google qui m'envoie : https://faq.vestiairecollective.com/hc/fr/articles/360002059098Quels-sont-les-avantages-de-I-option-Envoi-Direct-pour-les-vendeurs-J'ai la réponse à la question. Idem pour Vendeur Recommandé, j'ai la réponse à mon interrogation. 